\title{
Simple gene transfer technique based on I-SceI meganuclease and cytoplasmic injection in IVF bovine embryos
}

\author{
R.J. Bevacqua ${ }^{\text {a }}$, N.G. Canel ${ }^{\text {a }}$, M.I. Hiriart ${ }^{\text {a }}$, P. Sipowicz ${ }^{\text {b }}$, G.T. Rozenblum ${ }^{\text {c }}$, A. Vitullo ${ }^{\text {c }}$, \\ M. Radrizzani ${ }^{b}$, R. Fernandez Martin ${ }^{a}$, D.F. Salamone ${ }^{\mathrm{a}, *}$

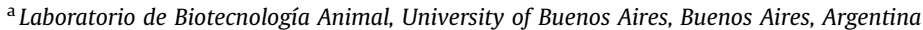 \\ ${ }^{\mathrm{b}}$ Laboratorio de Neuro y Citogenética Molecular, University of San Martín, Buenos Aires, Argentina \\ ${ }^{\mathrm{c}}$ Centro de Estudios Biomédicos, Biotecnológicos, Ambientales y de Diagnóstico, University of Maimónides, Buenos Aires, Argentina
}

\section{A R T I C L E I N F O}

\section{Article history:}

Received 29 August 2012

Received in revised form 8 March 2013

Accepted 8 March 2013

\section{Keywords:}

Exogenous DNA

gfp

Mammalian embryos

Mosaicism

\begin{abstract}
A B S T R A C T
Although transgenic methods in mammals are inefficient, an easy and highly efficient transgenesis system using I-Scel meganuclease (intron-encoded endonuclease from $S$. cerevisiae) was recently described in Xenopus. The method consisted of injection into fertilized eggs of an I-SceI reaction mixture with a plasmid DNA carrying the transgene, flanked by the meganuclease recognition sites (pIS). In the present study, the effects of I-Scel on gene transfer were tested apparently for the first time in mammals, in particular, in cattle. Various conditions were evaluated, including three concentrations of the plasmid pIS Pax6egfp, carrying I-Scel recognition sites flanking egfp under Pax6 promoter and two injection times (before IVM and after IVF) of pIS CAGegfp, carrying I-Scel sites fanking egfp under CAG promoter. In addition, the quantity of transgene was measured using quantitative polymerase chain reaction, and presence of transgene signals was evaluated using fluorescence in situ hybridization analysis. Transgene expression rates were higher $(\mathrm{P}<0.05)$ for groups treated after $\operatorname{IVF}(79.1 \%, 91 / 115$ and $63.0 \%, 75 /$ $119)$ than before IVM $(32.6 \%, 31 / 95$ and $34.7 \%, 33 / 95)$, with and without I-SceI, respectively. Interestingly, injection with pIS plus I-Scel after IVF increased frequency $(\mathrm{P}<0.05)$ of nonmosaic transgene-expressing embryos (58.3\%, $42 / 72$ vs. $29.7 \%, 25 / 84)$ for pIS plus I-Scel and pIS alone. Based on fluorescence in situ hybridization analysis, injection with I-Scel increased $(P<0.05)$ the proportion of embryos with transgene signals in all blastomeres compared with pIS alone $(44.0 \%, 11 / 25$ vs. $6.9 \%, 2 / 29)$ for pIS plus I-SceI and pIS alone. In addition, transgene copy number was numerically higher for the group treated with pIS plus I-Scel compared with pIS alone. In conclusion, I-Scel gene transfer increased transgene signals in bovine embryos.
\end{abstract}

(c) 2013 Elsevier Inc. All rights reserved.

\section{Introduction}

Transgenic mammals are essential for basic and applied research, but the available techniques remain inefficient. Pronuclear microinjection for generation of transgenic mice has been well established for more than 30 years [1]. Nevertheless, this method has many drawbacks: injected sequences randomly insert as concatemers, they have variable expression depending on the site of integration,

\footnotetext{
* Corresponding author. Tel.: +54 114524 8000; fax: +54 1145148737. E-mail address: salamone@agro.uba.ar (D.F. Salamone).
}

and they generally result in production of mosaic animals $[2,3]$. Cloning techniques using genetically modified cells have also been employed for transgenic animal production and have resulted in transgenic cattle [4,5]. The main advantage of cloning with a modified cell line is that it allows targeted transgenesis [6]. However, the overall efficiency of the process remains low $[7,8]$.

The genetic engineering of transgene constructs with the aim of increasing the probability of integration has attracted more attention in recent years, after it was shown that recombinases, integrases, and meganucleases derived from lower organisms could also function in mammalian cells. Two main approaches to transgenesis have been introduced. 
The first approach, which requires previous manipulation of the animal genome, is based on sequence-specific recombinases [9] and integrases [10]. For this method, animals carrying a docking site capable of interacting with the incoming sequence must be previously produced. Afterward, a transgene vector containing a compatible site is coinjected with the enzyme or mRNA encoding the recombinase or integrase. Despite allowing targeted transgenesis directly in the zygote, these techniques introduce practical limitations that make them difficult to apply to mammals other than mice. The second novel transgenesis approach does not require previous modification of the genome; it is based on designed meganucleases (zinc finger nucleases and transcription activator-like effector nucleases) [11,12]. The designed meganucleases carry two domains; a DNA binding domain linked to a nonspecific DNA cleavage domain [11,12]. The advantage of this technique is that it can control the recognition specificity of arbitrarily chosen chromosomal sites, without previous manipulation of the genome [13]. However, accessibility to these nucleases is limited to only a few laboratories. Another approach that does not require previous modification of the host genome is based on transposons. This technique was introduced after development of transposons capable of acting in mammal cells [14]. In the past few years, transposon-mediated transgenesis was efficient for production of genetically modified livestock, namely swine [15-17] and chickens [18].

Alternative approaches are based on natural meganucleases, also known as homing endonucleases. One of these meganucleases, I-SceI [19] was first described approximately 30 years ago, as a sequence-specific endonuclease that recognized large ( $>12$ base pair) sequence sites [20]. Transgenesis using the I-Scel meganuclease was efficient for production of transgenic killifish Medaka [21], Xenopus [22,23], and newts [24]. This method simply consists of digestion of a transgene construct carrying I-Scel recognition sites flanking the transgene, and cytoplasmic injection of a digestion mixture into fertilized eggs. Transgene expression was documented; furthermore, it was transmitted through the germ line and also expressed in offspring [22]. Ogino et al. [22] and Casco-Robles et al. [24] reported that a high proportion of the Xenopus and newt embryos had a nonmosaic expression pattern, and also demonstrated that the number of transgene integration sites was usually one or two, and that copy numbers ranged from one to four. Unfortunately, the mechanisms underlying the great success of the I-SceI transgenesis technique remain unclear. It is presumed that continued binding of I-SceI to the digested ends of injected transgenes prevented concatemer formation and/or degradation of linear monomers [22].

We recently observed that injection of zygotes with linear transgenes improved transgene expression efficiency in cattle [25], as previously reported [26,27]. These observations, in addition to results obtained in Xenopus, newts, and fish for meganuclease transgenesis, prompted us to test this technique in mammals, in particular cattle. In the present study, the effects of two constructs and two injection times were evaluated. The integration status of the transgene was assessed using fluorescence in situ hybridization (FISH) and the amount of transgene in embryos produced by injection with transgene alone or with the digestion mixture was compared using quantitative polymerase chain reaction (qPCR).

\section{Materials and methods}

\subsection{Experimental design}

The experimental design is summarized in Figure 1. Briefly, in the first experiment, three pIS concentrations (15, 25 , and $50 \mathrm{ng} / \mu \mathrm{L}$ ) of pIS Pax6egfp, plasmid carrying I-SceI recognition sites flanking egfp under Pax6 promoter, were compared for digestion mixture $(15+, 25+, 50+)$ and transgene alone (15-, 25-, 50-) injection after IVF (four replicates). In the second experiment, pIS CAGegfp (plasmid carrying I-Scel recognition sites flanking egfp under CAG promoter) was employed, and two injection times were compared: injection into immature oocytes (immediately after oocyte collection, before IVM) and into fertilized zygotes (after IVF), for digestion mixture and transgene alone groups (four replicates). Development to blastocysts and transgene expression were evaluated. Embryos produced by injection after IVF with digestion mixture or with transgene alone were subjected to qPCR and to FISH analysis. For qPCR, the amount of transgene was quantified at 30 minutes, at 22 hours after injection and at the blastocyst stage (Day 7; Day $0=$ IVF). For FISH, embryos were cultured until Day 2 (or 3) and subsequently synchronized, fixed, and subjected to double FISH with a control pericentromeric bovine probe and with an enhanced green fluorescent protein (egfp) probe.

\subsection{Chemicals}

Except where otherwise indicated, all chemicals were obtained from Sigma Chemical Company (St. Louis, MO, USA).

\subsection{DNA construction}

The plasmid used in the first experiment was pIS Pax6egfp, kindly provided by Dr. E. Amaya (Faculty of Life Sciences, The University of Manchester) and Dr. H. Ogino (Nara Institute of Science and Technology) [22], produced by insertion of the fragment Xbal/NotI from plasmid coding for egfp under Pax-6 promoter pPax-6GFP [28] into a modified version of pBluescript (ISceI-pBSII SK+, a gift from Dr. Ogino) [22], which has I-SceI sites flanking its multicloning site. The other experiments used pIS CAGegfp, produced by insertion of the fragment PstI-Sall from pCX-EGFP, codifying egfp under the chimeric cytomegalovirus-IE-chicken $\beta$-actin enhancer-promoter (CAG) [29], into ISceI-pBSII SK+. A schematic figure of both constructs is shown (Fig. 2).

\subsection{Oocyte collection and in vitro maturation}

Bovine ovaries were collected from abattoirs and transported to the laboratory at $25{ }^{\circ} \mathrm{C}$ to $30^{\circ} \mathrm{C}$. Cumulus-oocyte complexes were aspirated with 21-gauge needles from follicles with a diameter of 2 to $5 \mathrm{~mm}$ into HEPES-buffered Tyrode's albumin lactate pyruvate (HEPES-TALP). Oocytes covered with at least three layers of granulosa cells were selected for IVM. The maturation medium was bicarbonate-buffered TCM-199 (31100-035; Gibco, Grand Island, NY, USA), containing 10\% fetal bovine serum (013/07; Internegocios, Caba, Argentina), $10 \mu \mathrm{g} /$ $\mathrm{mL}$ follicle stimulating hormone (NIH-FSH-P1; Folltropin; 

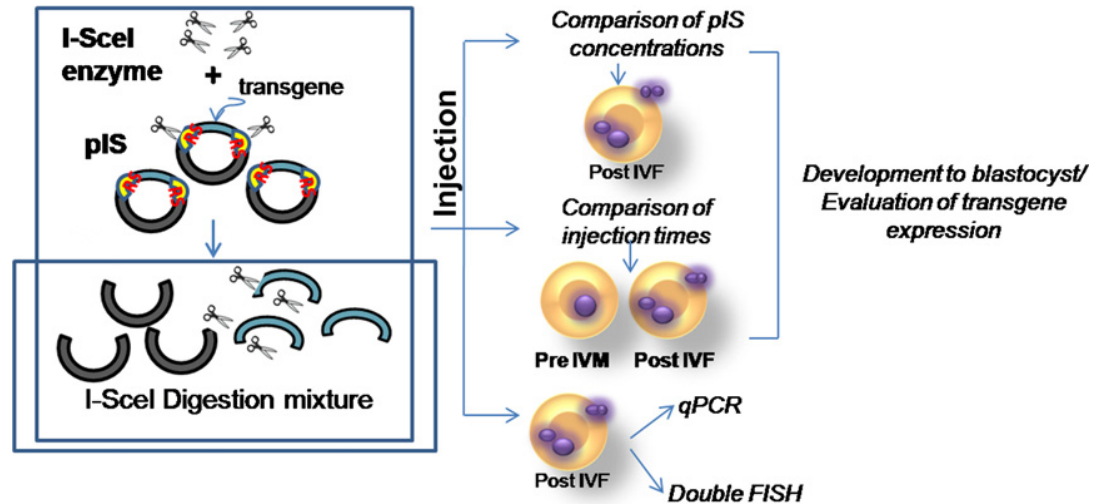

Fig. 1. Experimental design. In the first experiment, three transgene concentrations were tested for I-Scel or transgene-alone mediated transgenesis through injection into fertilized eggs. In the second experiment, injection into immature oocytes and fertilized eggs was compared for I-Scel transgenesis. In the third experiment, qPCR was used to determine transgene copy number in embryos. Finally, a FISH technique was used to detect transgene signals in embryos. FISH, fluorescence in situ hybridization, I-SceI, meganuclease; qPCR, quantitative polymerase chain reaction.

Bioniche, Caufield North, Victoria, Australia), $0.3 \mathrm{mM}$ sodium pyruvate (P2256), $100 \mu \mathrm{M}$ cysteamine (M9768), and 2\% antibiotic-antimycotic (15240-096; Gibco). The oocytes were incubated for 24 hours in mineral oil (M8410) in $100-\mu \mathrm{L}$ droplets, in $6.5 \% \mathrm{CO}_{2}$ in humidified air at $39^{\circ} \mathrm{C}$.

\subsection{IVF procedure}

Frozen semen was thawed in a $37{ }^{\circ} \mathrm{C}$ water bath for 30 seconds. Sperm were then centrifuged twice $(490 \times g$ for 5 minutes) in Brackett-Oliphant medium (BO) [30] and resuspended in BO supplemented with $5 \mathrm{mM}$ caffeine (C4144) and $20 \mathrm{IU} / \mathrm{mL}$ heparin (H3149). Sperm were adjusted to $40 \times 10^{6} /$ $\mathrm{mL}$ and diluted to half concentration $\left(20 \times 10^{6} / \mathrm{mL}\right)$ with $\mathrm{BO}$ containing $10 \mathrm{mg} / \mathrm{mL}$ fatty acid-free bovine serum albumin (A6003). The cumulus-oocyte complexes were washed twice with BO plus $5 \mathrm{mg} / \mathrm{mL}$ FAF-BSA (fatty acid- free bovine serum albumin) and subsequently exposed to the sperm suspension for 5 hours in a $100-\mu \mathrm{L}$ drop at $39{ }^{\circ} \mathrm{C}$ in $5 \% \mathrm{CO}_{2}$ in humidified air. Presumptive zygotes were then washed three times in HEPES-TALP, and, cumulus cells were removed by vortexing for 2 minutes in $1 \mathrm{mg} / \mathrm{mL}$ hyaluronidase (H-4272; in Dulbecco's PBS). Then, presumptive zygotes were washed in HEPES-TALP, selected by visualization of at least one polar body, and immediately injected with pIS digestion mixture or with pIS alone.

\subsection{Digestion mixture}

The restriction digestion of pIS Pax6egfp or pIS CAGegfp with I-Scel meganuclease was carried out at $37{ }^{\circ} \mathrm{C}$ for 40 minutes before injection into fertilized or immature oocytes. The digestion mixture consisted of $1 \times$ I-SceI buffer, $0.5 \mathrm{U} / \mu \mathrm{L}$ I-SceI (R0694S, New England Biolabs, Ipswich, MA, USA), $500 \mathrm{ng} / \mu \mathrm{L}$ of pIS Pax6egfp or pIS CAGegfp and water to reach a final $20-\mu \mathrm{L}$ volume and a final $50-\mathrm{ng} / \mu \mathrm{L}$ transgene
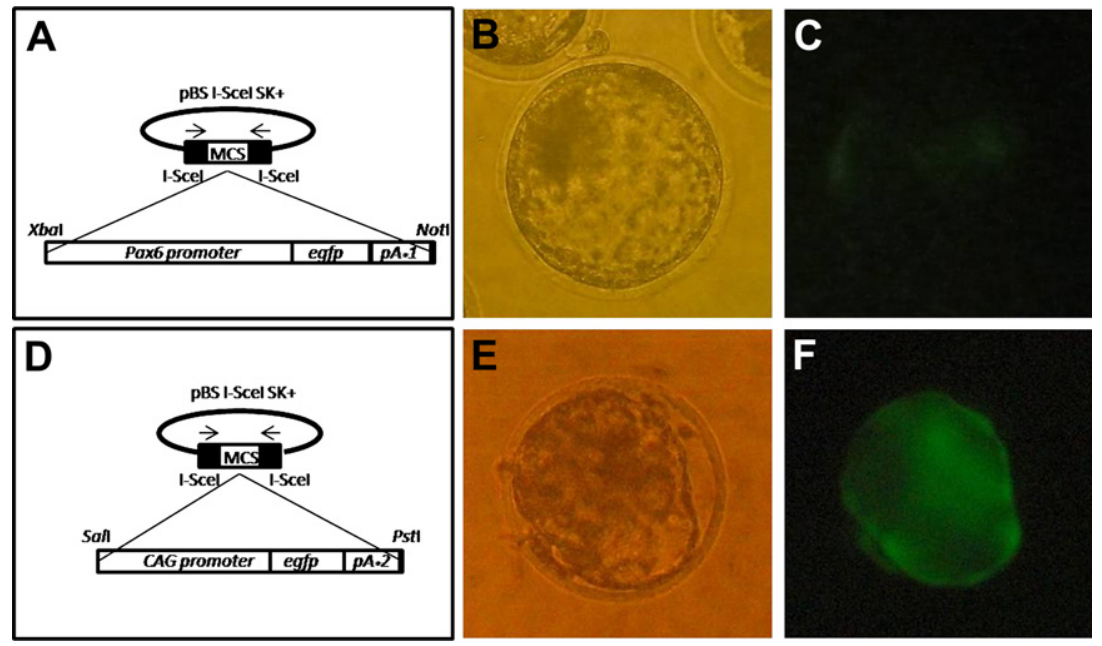

Fig. 2. Comparison of pIS Pax6egfp and pIS CAGegfp expression in bovine blastocysts. (A) pIS Pax6egfp construct; (B) and (C) blastocyst produced by I-SceI mediated transgenesis with 50 ng/uL pIS Pax6egfp; (D) pIS CAGegfp construct; (E) and (F) blastocyst produced by I-SceI mediated transgenesis with 50 ng/uL pIS CAGegfp. (B) and (E) Under bright light. (C) and (F) Under blue light (488 nm). pA: polyadenilation site. In A), pA $\times 1$ : SV40 pA; in D), pA $\times 2$ : rabbit $\beta$ globin pA. CAGegfp, egfp under CAG promoter; I-SceI, meganuclease; MCS, multiple cloning site; Pax6egfp, egfp under Pax6 promoter; pBS, pBluescript; pIS, plasmid with I-Scel recognition sites flanking the transgene. 
concentration. After digestion, $0.8 \%$ agarose gel electrophoresis was performed to verify digestion of the construct.

\subsection{Injection of transgene digestion mixture with I-SceI or transgene alone}

Immature and fertilized oocytes were subjected to hyaluronidase treatment, then transferred to $20-\mu \mathrm{L}$ droplets of HEPES-TALP and injected, using a $9-\mu \mathrm{m}$ pipette, with $10 \%$ Polyvinylpyrrolidone containing $50 \mathrm{ng} / \mu \mathrm{L}$ of: pIS Pax6egfp, pIS Pax6egfp digested with I-SceI, pIS CAGegfp, or pIS CAGegfp digested with I-SceI in a volume $<10 \mathrm{pL}$. Immature oocytes were matured in vitro after injection, as described previously herein. The zygotes were then subjected to IVF and cultured as described in the next section.

\subsection{In vitro embryo culture}

Presumptive zygotes were cultured in $50-\mu \mathrm{L}$ droplets of Synthetic oviductal fluid medium [31] supplemented with $2.5 \%$ fetal bovine serum at $39{ }^{\circ} \mathrm{C}$ in $6.5 \% \mathrm{CO}_{2}$ in humidified air. Embryos were transferred to a new droplet every 48 hours. Cleavage was evaluated on Day 2 and the number of blastocysts on Day 7 post IVF.

\subsection{Evaluation of EGFP fluorescence in embryos}

All the embryos produced were briefly exposed to blue light using an excitation filter at $488 \mathrm{~nm}$ and an emission filter at $530 \mathrm{~nm}$ to determine egfp expression. Embryos were evaluated on Days 4 and 7 after IVF.

\subsection{Real-time polymerase chain reaction ( $q P C R)$}

Embryos produced using injection after IVF with pIS CAGegfp digestion mixture or with pIS CAGegfp alone were transferred as $1-\mu \mathrm{L}$ aliquots into Eppendorf tubes, resuspended in $9 \mu \mathrm{L}$ of $1 \times$ extraction polymerase chain reaction (PCR) buffer (jump PCR completer) with proteinase $\mathrm{K}$ ( $1 \mathrm{mg} / \mathrm{mL}$; V302B; Promega, Madison, WI, USA) and incubated at $56{ }^{\circ} \mathrm{C}$ for 1 hour and at $95{ }^{\circ} \mathrm{C}$ for 10 minutes. Thereafter, qPCR was performed using a Stratagene MX305p (Agilent Technologies), on a final volume of $25 \mu \mathrm{L}$. Reagents employed were $2 \times$ SYBR Green PCR master mix (4309155; Applied Biosystems), $0.04 \mu \mathrm{M}$ Rox dye, $0.1 \mu \mathrm{M}$ primer forward oGFPq1F (CACATGAAGCAGCACGACTT), $0.1 \mu \mathrm{M}$ primer reverse oGFPq2R (GGTCTTGTAGTTGCCGTCGT), and $1 \mu \mathrm{L}$ of proteinase K-treated embryo DNA. Cycling conditions were: 1 cycle at $95^{\circ} \mathrm{C}$ for 10 minutes, followed by $95^{\circ} \mathrm{C}$ for 30 seconds and $58^{\circ} \mathrm{C}$ for 40 seconds during 60 cycles, and a final extension at $72{ }^{\circ} \mathrm{C}$ for 20 seconds. Afterward, a dissociation curve was included up to $95^{\circ} \mathrm{C}$. The PCR efficiency calculated from slope was $R^{2}$ (qPCR 1): $0.992 ; R^{2}(\mathrm{qPCR} 2): 0.943$. Data were analyzed using Stratagene MX3005P V4.10, including three replicates for each sample. The dose-response curve for the qPCR was calculated using qPCR measurement on five known dilutions of the pIS CAGegfp construct (3000, 300, 30,3 , and $0.3 \mathrm{fg}$ ). The trendland equation that best fitted the curve was: number of molecules $=2 \mathrm{E}+10 \times \mathrm{e}-0.53 \mathrm{Ct}$, $R^{2}=0.969$. The number of copies of the transgene was calculated considering $600 \times 6 \times 10^{3}$ the molecular weight of the plasmid.

\subsection{Fluorescence in situ hybridization}

Days 2 and 3 embryos produced by injection after IVF with pIS CAGegfp digestion mixture with I-Scel or with pIS CAGegfp alone were subjected to FISH using a pCX- EGFP plasmid sequence and a chromosome 19 pericentromeric bovine sequence as probes. Embryos were incubated for 6 hours with $0.1 \mu \mathrm{g} / \mathrm{mL}$ demecolcine (D1925). Afterward, embryos were treated with pronase, placed on slides, and fixed with 3:1 methanol-acetic acid. The pCX-EGFP was labeled with Rhodamine-5-2'-Deoxyuridine, 5'-Triphosphate (RU-013-0135; eENZYME, Montgomery Village, MD, USA) using the Nick Translation System (18156-010; Invitrogen). To build a chromosome 19 control probe, the bacterial artificial chromosome $\mathrm{CH} 24050011$ (http://bacpac.chori.org/ bovine240.htm) was labeled with Fluorescein-12-2'-Deoxyuridine, 5'-Triphosphate (RU-013- 0125; eENZYME) using the the Nick Translation System. The DNA probes were denatured for 10 minutes at $85{ }^{\circ} \mathrm{C}$ in a hybridization mix (H.Mix) containing 50\% formamide (Merck, Darmstadt, Germany), 40\% sulfate dextrane (D8906), 20× sodium chloride-sodium citrate (SSC) $10 \%$ and $0.03 \%$ herring sperm DNA. The proportion used was 7:1:1 (H.Mix:labeled pCXEGFP:control probe). Before application of the denatured probe, fixed embryos on slides were denatured with $70 \%$ formamide in $2 \times$ SSC for 2 minutes at $72{ }^{\circ} \mathrm{C}$ and dehydrated by successive passage through $70 \%, 95 \%$, and $100 \%$ ethanol. The probe was incubated overnight in a moist dark chamber at 37 ${ }^{\circ} \mathrm{C}$. After incubation, coverslips were detached with a short incubation in $2 \times \mathrm{SSC}$, the slides were washed at $72{ }^{\circ} \mathrm{C}$ for 2 minutes in $0.4 \times$ SSC with $0.3 \%$ Tween and then washed at room temperature in $2 \times$ SSC with $0.1 \%$ Tween. Total DNA was counterstained with DAPI (4',6-Diamidino-2-Phenylindole, Dilactate) and coverslips were reapplied. Images of each cell and their signals were recorded with an Optronics camera.

\subsection{Statistical analyses}

In vitro embryo development and fluorescent expression were compared using Fisher Exact test, whereas qPCR results were analyzed using a Kruskal-Wallis test. All analyses were conducted using SAS [32] and differences were considered significant when $\mathrm{P}<0.05$.

\section{Results}

\subsection{Experiment 1: Comparison of three pIS Pax6egfp} concentrations for I-SceI- and transgene alone-mediated gene transfer

In the first experiment, three pIS Pax6egfp concentrations were compared for I-Scel- and transgene alone-mediated gene transfer. The three concentrations evaluated yielded similar blastocysts rates. However, except the groups injected with 25 and $50 \mathrm{ng} / \mu \mathrm{L}$ digested by I-SceI, treatments reduced $(\mathrm{P}<0.05)$ blastocyst rates compared with the IVF control (Table 1 and Fig. 2). Although transgene expression rates at Day 4 were higher for the group injected with $50 \mathrm{ng} / \mu \mathrm{L}$ pIS 
Table 1

Comparison of three pIS Pax6egfp concentrations for I-Scel and transgene alone-mediated transgenesis in bovine IVP embryos.

\begin{tabular}{|c|c|c|c|c|c|c|}
\hline \multirow[t]{2}{*}{ pIS Pax6egfp (ng/ $\mu \mathrm{L})$} & \multirow[t]{2}{*}{ I-SceI $(0.5 \mathrm{U} / \mu \mathrm{L})$} & \multirow[t]{2}{*}{$\mathrm{N}$} & \multirow[t]{2}{*}{ Cleavage (\%) } & \multirow[t]{2}{*}{ Blastocysts (\%) } & \multicolumn{2}{|c|}{ Egfp expression (\%) } \\
\hline & & & & & $\overline{\mathrm{D} 4}$ & $\overline{\text { Blastocysts }}{ }^{\mathrm{a}}$ \\
\hline 0 & - & 80 & $76(95.0)^{b, c}$ & $31(38.7)^{b}$ & $0(0)$ & $0(0)$ \\
\hline \multirow[t]{2}{*}{15} & + & 69 & $57(82.6)^{\mathrm{d}}$ & $13(18.8)^{d}$ & $4(5.7)^{\mathrm{d}}$ & $2(15.3)$ \\
\hline & - & 98 & $90(91.8)^{b, d}$ & $22(22.4)^{\mathrm{d}}$ & $1(1.0)^{\mathrm{d}}$ & $5(22.7)$ \\
\hline \multirow[t]{2}{*}{25} & + & 89 & $86(96.6)^{b}$ & $25(28.0)^{b, d}$ & $3(3.3)^{d}$ & $5(20.0)$ \\
\hline & - & 91 & $81(89.0)^{b, d}$ & $19(20.8)^{\mathrm{d}}$ & $4(4.3)^{d}$ & $8(42.1)$ \\
\hline \multirow[t]{2}{*}{50} & + & 80 & $69(86.2)^{\mathrm{c,d}}$ & $22(27.5)^{\mathrm{b}, \mathrm{d}}$ & $1(1.2)^{d}$ & $8(36.3)$ \\
\hline & - & 71 & $61(85.9)^{d}$ & $16(22.5)^{d}$ & $19(26.7)^{\mathrm{b}}$ & $8(50.0)$ \\
\hline
\end{tabular}

Day 0: day of IVF and injection.

Abbreviations: D4, Day 4; I-SceI, meganuclease; IVP, in vitro production; Pax6egfp, egfp under Pax6 promoter; pIS, plasmid with I-SceI recognition sites flanking the transgene.

a egfp blastocysts were calculated as a percentage of total blastocysts.

b-d Within a column, means without a common superscript differed $(\mathrm{P}<0.05)$.

Pax6egfp alone, at the blastocyst stage, there were no significant differences between I-Scel and transgene-alone gene transfer. Transgene-expressing blastocyst rates were numerically higher for the groups injected with $50 \mathrm{ng} / \mu \mathrm{L}$.

\subsection{Experiment 2: Comparison of two injection times (before} IVM and after IVF) for I-SceI- and transgene alone-mediated gene transfer

In the second experiment, pIS CAGegfp was used at $50 \mathrm{ng} / \mu \mathrm{L}$. In this construction, egfp was under a strong and early expression promoter, well known in mammals.
Cytoplasmic injection was evaluated before IVM and after IVF. For both injection times, transgene expression was detected, however, embryo development was lower for immature oocytes $(\mathrm{P}<0.05)$. Despite observing higher egfp expression rates on Day 4 for I-Scel gene transfer after IVF, egfp-expressing blastocysts did not differ between transgene alone or digested by I-SceI (Fig. 3 and Table 2). For the group injected after IVF, mosaic expression at Day 4 was determined for a group of embryos. Homogeneous expression was detected in $58.3 \%$ of the embryos that expressed the transgene (42/72) in the group injected with the digestion mixture; this was higher than for the group
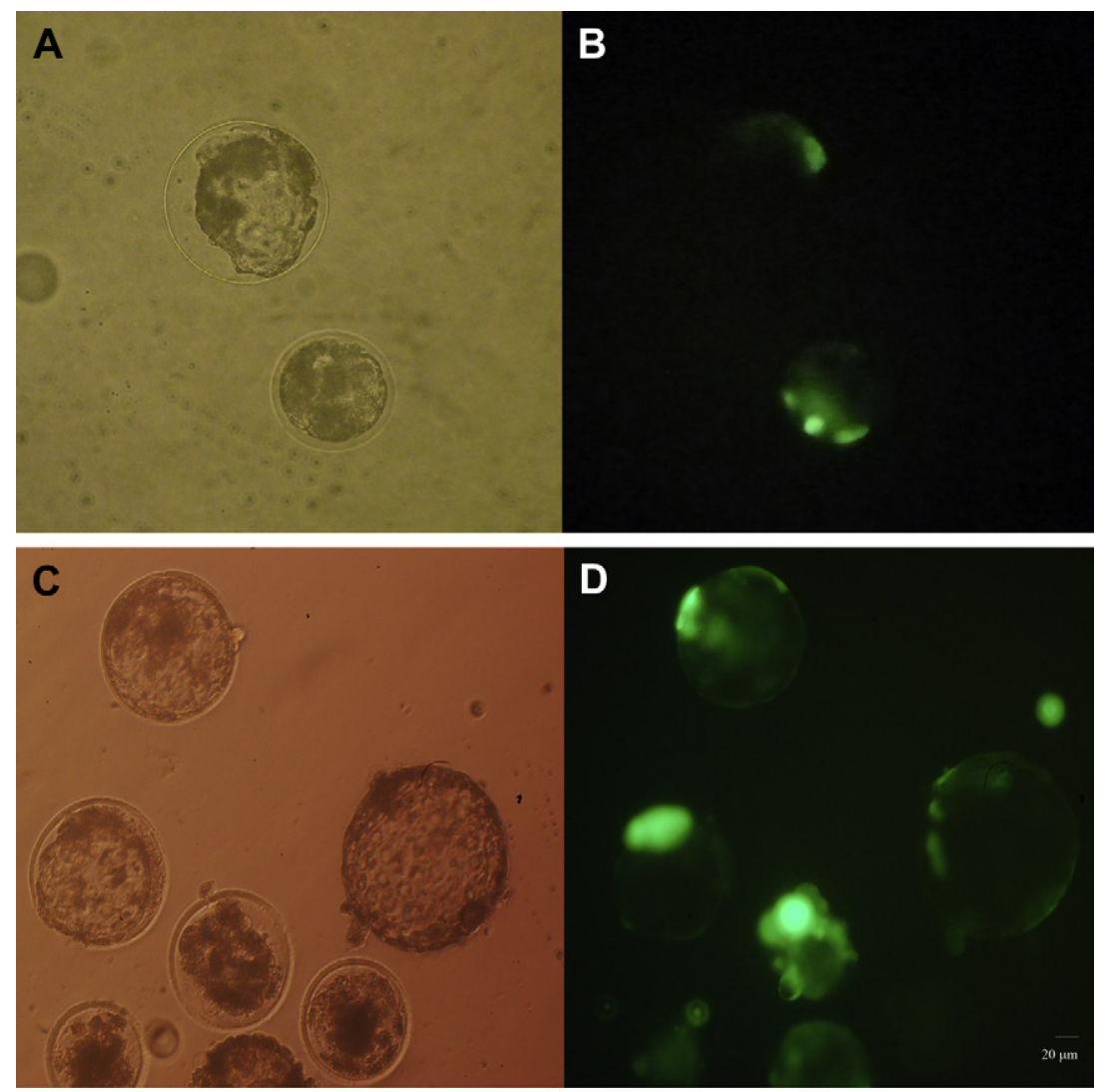

Fig. 3. Bovine egfp-expressing blastocysts produced using I-Scel transgenesis through injection into (A) and (B) immature oocytes (bright light); and (C) and (D) fertilized oocytes (blue light; $488 \mathrm{~nm}$ ). I-SceI, meganuclease. 
Table 2

Comparison of injection time (before IVM and after IVF) for I-SceI and transgene alone-mediated transgenesis in bovine IVP embryos.

\begin{tabular}{|c|c|c|c|c|c|c|}
\hline \multirow[t]{2}{*}{ pIS CAGegfp injection time } & \multirow[t]{2}{*}{ I-SceI $(0.5 \mathrm{U} / \mu \mathrm{L})$} & \multirow[t]{2}{*}{$\mathrm{N}$} & \multirow[t]{2}{*}{ Cleavage (\%) } & \multirow[t]{2}{*}{ Blastocysts (\%) } & \multicolumn{2}{|c|}{ egfp expression (\%) } \\
\hline & & & & & $\overline{\mathrm{D} 4}$ & Blastocysts $^{\mathrm{a}}$ \\
\hline \multirow[t]{3}{*}{ Before IVM } & + & 95 & $70(73.6)^{b}$ & $4(4.2)^{b}$ & $31(32.6)^{b}$ & $3(75.0)^{b}$ \\
\hline & - & 95 & $68(71.5)^{b}$ & $3(3.1)^{b}$ & $33(34.7)^{\mathrm{b}}$ & $2(66.0)^{\mathrm{b}}$ \\
\hline & IVF control $^{\mathrm{C}}$ & 119 & $66(55.4)^{d}$ & $16(13.4)^{\mathrm{d}}$ & $0(0)$ & $0(0)$ \\
\hline \multirow[t]{3}{*}{ After IVF } & + & 115 & $110(95.6)^{\mathrm{e}}$ & $40(34.7)^{\mathrm{e}}$ & $91(79.1)^{\mathrm{d}}$ & $32(80.0)^{\mathrm{b}}$ \\
\hline & - & 119 & $107(89.9)^{\mathrm{e}}$ & $46(38.6)^{\mathrm{e}}$ & $75(63.0)^{\mathrm{e}}$ & $35(76.0)^{b}$ \\
\hline & IVF control & 217 & $201(92.6)^{\mathrm{e}}$ & $84(38.7)^{\mathrm{e}}$ & $0(0)$ & $0(0)$ \\
\hline
\end{tabular}

Abbreviations: CAGegfp, egfp under CAG promoter; D4, Day 4; I-SceI, meganuclease; IVP, in vitro production; pIS, plasmid with I-SceI recognition sites flanking the transgene.

a egfp blastocysts and their respective statistic were calculated as a percentage of total blastocysts.

b,d,e Within a column, means without a common superscript differed $(\mathrm{P}<0.05)$.

c This IVF control was denuded before IVM, then IVM and 22 hours later subjected to IVF.

injected with transgene alone, for which only $29.7 \%(25 / 84)$ embryos had nonmosaic transgene expression $(P<0.05)$. When the injection was performed into immature oocytes, it was difficult to determine mosaicism rates, because many embryos had already begun fragmentation at Day 4 .

\subsection{Experiment 3: Quantification of transgene in embryos} produced by I-SceI-mediated gene transfer using real time polymerase chain reaction ( $q P C R$ )

The plasmid used in this study has no mammalian origin of replication. Therefore, only integration into the genome could increase the amount of transgene during embryo development to the blastocyst stage. The amount of transgene present in embryos injected with I-Scel digestion mixture or with transgene alone was quantified by realtime PCR at 30 minutes and at 22 hours after injection, and at the blastocyst stage. No statistical differences were detected either between different embryo stages within one particular treatment or between treatments at the same embryo stage. However, at 30 minutes after injection and also at the blastocyst stage, the group injected with I-Scel digestion mixture exhibited more transgene (media transgene copy number \pm SEM: $25620 \pm 8940 ; 2250 \pm$ $525 ; 6540 \pm 3345$, for quantification at 30 minutes, 22 hours, and the blastocyst stage, respectively) compared with the transgene alone group (media transgene copy number \pm SEM: $4320 \pm 960 ; 1185 \pm 360$; and $2250 \pm 870$, for quantification at 30 minutes, 22 hours, and the blastocyst stage, respectively; Fig. 4).

\subsection{Experiment 4: FISH of embryos produced by I-SceI and transgene alone-mediated gene transfer}

Although the FISH technique is quite inefficient in embryos, 54 embryos were evaluated (those lacking control probe signals were discarded). There were more transgenepositive blastomeres detected for the group injected with I-Scel digestion mixture than for the group injected with transgene alone $(79.4 \%, 54 / 68$ for I-Scel vs. $46.9 \%, 38 / 81$ for transgene alone gene transfer, $\mathrm{P}<0.05$ ). In addition, for the group injected with I-SceI, more embryos $(\mathrm{P}<0.05)$ had transgene signals in all blastomeres (Table 3 ). Furthermore, the total number of embryos with at least one transgene signal was also determined; it was noteworthy that there were no significant differences between I-Scel and transgene-alone groups (24/25 vs. 21/29 embryos with at least one transgene signal for the I-Scel and transgene-alone groups, respectively). In that regard, there was a higher proportion of embryos with transgene signals in all of their blastomeres for the group injected with the digestion mixture compared with the transgene alone $(\mathrm{P}<0.05$; Table 3 and Fig. 5). The number of transgene signals in blastomeres ranged from 1 to 4 .

\section{Discussion}

The method used in this study was characterized by its simplicity: injecting fertilized eggs with a reaction mixture of I-Scel meganuclease and a plasmid DNA carrying a transgene cassette flanked by I-Scel recognition sites. Reports in amphibians such as Xenopus [22,32] and newts [24], and also in fish [21,33] described the high efficiency and simplicity of I-SceI meganuclease-mediated gene transfer. However, to date this technique has apparently not been tested in mammals. In the present study, transgene-expressing bovine embryos were produced using I-Scel gene transfer.

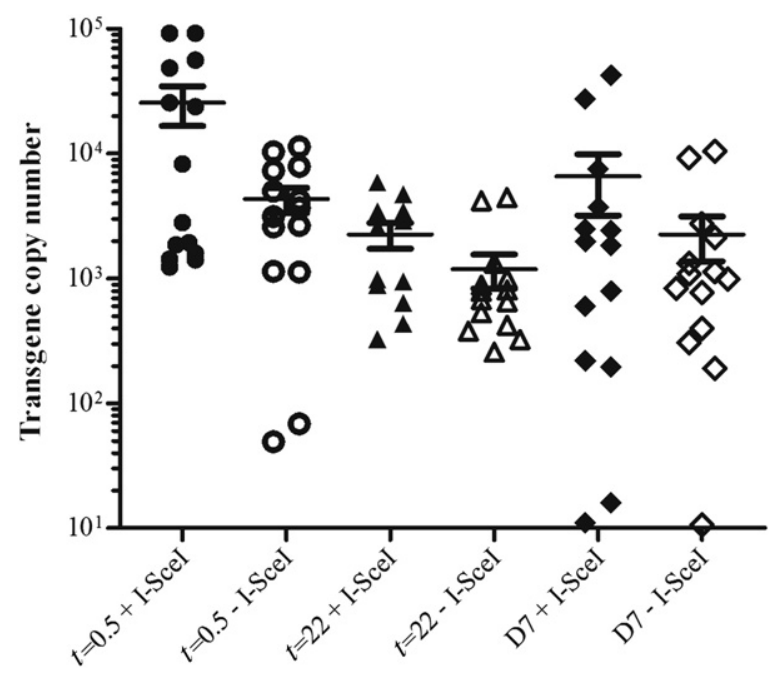

Fig. 4. Quantification of transgene (number of copies of the transgene) using real time polymerase chain reaction at 0.5 hours, 22 hours, and Day 7 (blastocyst stage) for meganuclease (+ I-SceI) or transgene alone (- I-SceI) transgenesis. I-SceI, meganuclease. 
Table 3

Fluorescence in situ hybridization analysis of bovine embryos produced by I-Scel or transgene alone-mediated transgenesis.

\begin{tabular}{|c|c|c|c|c|c|c|c|}
\hline \multirow[t]{2}{*}{ Group I-SceI } & \multirow[t]{2}{*}{ Embryos, $\mathrm{N}$} & \multirow[t]{2}{*}{ Blastomeres, N } & \multirow[t]{2}{*}{ Blastomeres, $\operatorname{tg}+(\%)$} & \multicolumn{3}{|c|}{ Embryos (\%) } & \multirow{2}{*}{$\frac{\text { Cr19 probe (\%) }}{\text { euploid }}$} \\
\hline & & & & $\overline{\operatorname{tg}+{ }^{a}}$ & $\operatorname{tg}-$ & nd & \\
\hline+ & 25 & 68 & $54(79.1)^{b}$ & $11(44.0)^{b}$ & $1(4.0)^{b}$ & $7(28.0)$ & $21(84.0)$ \\
\hline- & 29 & 81 & $38(46.9)^{c}$ & $2(6.9)^{c}$ & $8(27.8)^{c}$ & $5(17.2)$ & $22(75.8)$ \\
\hline
\end{tabular}

$\operatorname{tg}+$ indicates presence of the transgene signal, and tg- indicates absence of the transgene signal.

Abbreviations: +, injected with digestion mixture with I-SceI; -, injected with transgene alone; Cr19 probe, bovine chromosome 19 pericentromeric probe; I-SceI, meganuclease; nd, not determined (embryos showing only one analyzable blastomere).

a Embryos showing transgene signal in all of their blastomeres.

b,c Within a column, means without a common superscript differed $(\mathrm{P}<0.05)$.

Furthermore, it was noteworthy that this technique also resulted in an increased proportion of embryos with transgene signals in all of their blastomeres. In addition, we concluded that injection can be performed into immature oocytes or presumptive zygotes, resulting in production of transgene-expressing embryos.

In the first experiment, I-SceI-mediated gene transfer was tested with a construct carrying I-SceI recognition sites flanking a Pax6gfp, gfp under Pax6 promoter, sequence. The Pax 6 promoter had apparently never been used to induce transgene expression in bovine embryos. The Pax 6 transcription factor, which acts high in the regulatory hierarchy, has been described as fundamental for eye and lens development in humans, mice, zebrafish, and Drosophila [34-37]. Based on this first experiment, we inferred that the Pax 6 promoter can induce leaky expression of the egfp gene from early activation of the bovine embryo genome at Day 4 [38]. In addition, injecting meganuclease did not affect embryo viability. Although no differences in transgene-expressing blastocysts were detected between digested and undigested pIS Pax6egfp, egfp-expressing blastocysts were numerically higher for the $50 \mathrm{ng} / \mu \mathrm{L}$ concentration; therefore, that concentration was used for the following experiments.

In subsequent experiments, a well characterized, strong CAG promoter (the chimeric cytomegalovirus-IE-chicken $\beta$-actin enhancer-promoter) was used. With this construct, there was an increased proportion of nonmosaic egfp embryos at Day 4 for the group injected with I-SceI after IVF than for the group injected with the transgene alone. In previous reports in amphibians and fish, there was also higher transgenesis and homogeneous expression rates after digestion mixture injection [21,22,39]. One of the main concerns to achieve integration of the transgene through the I-Scel transgenesis technique, as noted by Ogino et al. [22] and Thermes et al. [21], was to inject the ISceI reaction as soon as possible after egg fertilization. In our study, this was achieved through transgene injection before IVM or immediately after IVF. We had previously reported that injection of the transgene into metaphase II oocytes before IVF resulted in poor cleavage and subsequent embryo development, possibly because of premature cortical granule exocytosis in response to the injection [40]. Consequently, in the present study, injection into immature oocytes was done in an attempt to avoid that problem. Although it was possible to produce transgene-expressing bovine embryos by injecting exogenous DNA into immature oocytes, injecting DNA after IVF resulted in higher embryo development rates. Interestingly, egfp/total blastocyst rates were not significantly different between the two injection times, indicating that early injection affected embryo viability but not transgene expression efficiency.
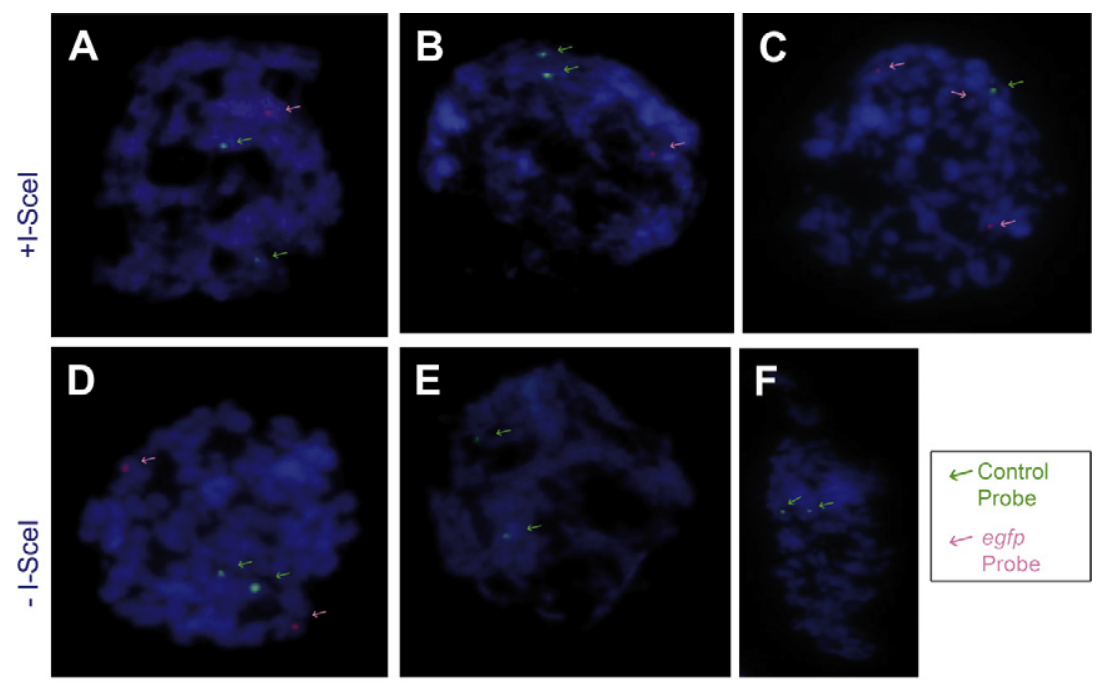

Fig. 5. Detection of transgene signals using FISH for (A), (B), and (C) I-SceI transgenesis and for (D), (E), and (F) transgene-alone transgenesis. Pink arrows indicate transgene signals (rhodamine-labeled probe) and green arrows indicate bovine chromosome 19 pericentromeric signals (FITC-labeled probe). Additional figures are shown in the Supplementary File. FISH, fluorescence in situ hybridization; FITC, Fluorescein isothiocyanate; I-SceI, meganuclease. 
The poor development rates for the groups injected before IVM seemed to be related to the lack of cumulus cells during maturation. It is well known that the presence of the cumulus cells is crucial for nuclear and cytoplasmic maturation [41]; consequently, removal of cumulus cells before injection and to IVM, reduced embryo viability. That injection of the transgene before IVM can result in transgene expression up to the blastocyst stage, could be very useful for the study of early maturation events, through injection of interference RNAs, exogenous DNAs, or mRNAs. Although this strategy has apparently never been tested in cattle, it has been done in chickens and mice $[42,43]$. Future research should be directed to enhance the viability of injected oocytes with only a few layers of cumulus cells.

For groups injected after IVF, which resulted in the highest blastocysts and egfp expression rates, the quantity of transgene at 0.5 hours, 22 hours, and Day 7 (blastocyst stage) after injection was measured using qPCR and the number of molecules of transgene present was calculated using a highly sensitive dose-response curve. Although the transgene copy numbers did not differ among groups, the quantity of transgene present shortly after injection was numerically higher for the group injected with the I-Scel digestion mixture. In addition, the quantity of transgene at the blastocyst stage was higher than observed at 22 hours after injection, especially for the group injected with I-Scel digestion mixture. Because the exogenous DNA employed in this work had no mammalian origin of replication, this could only be explained by integration. In contrast to restriction enzyme mediated integration transgenesis, in which transgene integration is stimulated by partial digestion of the host genome by coinjected restriction enzymes [44], in IScel transgenesis, the host genome does not contain the known I-Scel recognition site. Choulika et al. [45] reported that digestion by I-Scel provoked the insertion product of interest to remain as short fragments, exposing more recombinogenic ends. Through association with one of its cleavage products, I-Scel might accomplish 'inhibition' of endogenous ligases/replicases by digestion of generated concatemers, or by protection of digested recombinogenic ends from degradation, ligation, or both. This protective action of I-Scel could explain the high quantity of transgene detected at 0.5 hours after injection, as proposed by Thermes et al. [21], for meganuclease transgenesis in fish. Persistence of ectopic plasmids in blastocysts could also indicate that even in the case of digestion mixture injection, sometimes the meganuclease does not digest on both sides of the transgene, allowing concatemer formation and persistence of ectopic plasmids. If the transgene integrated only once in the genome, approximately 150 copies of transgene would be expected at the blastocyst stage. However, quantities of transgene higher than expected as a result of integration, were detected for transgene plus meganuclease and for transgene alone. Consequently, we propose the following scenarios: (1) integration of transgene molecules as concatemers; (2) more than one copy of transgene integrated which (combined with 1 ) resulted in an important range of measures; and (3) the presence of ectopic plasmids even at the blastocyst stage, as reported [46].

Beause the main interest in mammal transgenesis is to assure incorporation of the transgene into the genome, in a subsequent experiment we performed FISH of preimplantation embryos (for groups injected after IVF). This is the most powerful tool available to detect in situ presence of a transgene and the sole option available to detect transgene signals in preimplantatory embryos. Unfortunately, the low amount of DNA present in a blastocyst did not enable the use of other techniques, such as Southern blot analysis. Therefore, in this work, more than 50 embryos were analyzed, using a control bovine chromosome 19 pericentromeric probe and a pCX-EGFP probe (with $90 \%$ homology to the pIS CAGegfp sequence). This experiment provided conclusive evidence that I-Scel-mediated gene transfer increased the proportion of embryos showing transgene signals in all of their blastomeres (refer to the Supplementary File for visualization of a higher number of blastomeres). Total numbers of embryos showing transgene signal in at least one of their blastomeres did not differ between transgene alone and transgene plus meganuclease groups; however, I-SceI-mediated gene transfer increased the proportion of blastomeres with transgene signals per embryo. Perhaps mosaic expression of green fluorescent protein reflected persistence of injected plasmids as extrachromosomal episomes which are inherited only by a subset of cells $[47,48]$ and also to late integration in the genome. This phenomenon was frequently observed after pronuclear microinjection and intracytoplasmic sperm injection-mediated gene transfer in mammals [26,27], and it continues to be one of the main limitations of most of the current transgenesis techniques in mammals. These ectopic plasmids are eliminated during the steps before FISH hybridization; therefore, the increased proportion of embryos with a transgene signal in all of their blastomeres observed in this work for I-SceI gene transfer in the bovine could have been the result of increased frequency of integration at the one-cell stage, as proposed by Thermes et al. [21] for fish. These observations could also explain the controversial results obtained using qPCR and FISH. The high transgene copy numbers detected with qPCR were because of the presence of integrated and episomal transgenes. As the meganuclease binds to the ends of the linear fragments generated by its digestion, it protects them from degradation and from concatemer formation, and higher transgene copy numbers are detected. Conversely, we inferred that incubation in demecolcine before FISH provoked condensation of the chromatin and rupture of the nuclear envelope, resulting in loss of most episomal DNA during washing (only large concatemers persisted after washing). Then, as the meganuclease avoided concatemer formation, our hypothesis is that the increased transgene signals observed for I-Scel gene transfer were related to integration and not to persistence of episomal plasmids. In support of the previous statement, Thermes et al. [21] also reported that transgene integration never occur as long concatemers, reducing the gene-silencing effect frequently observed in vertebrates [49]. Instead, the transgene was integrated in short repeats and consequently not silenced, which could have a great effect on mammalian transgenesis technology.

Based on the evidence presented so far, we inferred that the action of I-Scel on the genome was indirect. The meganuclease introduced highly recombinogenic double 
stranded breaks at both ends of the insert of interest, and protected it from degradation. In the context of the early zygote environment, in which DNA repair machinery is active [25], these double-stranded breaks of the transgene are joined to similar breaks in the genome by recombination reactions. A remarkable aspect of the I-SceI gene transfer technique is that the meganuclease should not directly digest the genome, because no canonical 18-base pair I-SceI restriction site was detected using blastn (NCBI program that searches nucleotide databases using a nucleotide query) in the bovine genome. However, it is possible that the binding of the meganuclease to the transgene fragments stimulated recruitment of the repairing proteins. The amplified activity of the DNA repair machinery, the high number of ruptures in the genome (exacerbated by the transgene injection), and the presence of many linear transgene fragment substrates for recombination seemed to be responsible for the increased presence of transgene signals for this technique.

\subsection{Conclusions}

This was apparently the first report regarding the use of I-Scel gene transfer in a mammalian species, namely cattle. Not only was this technique simple and versatile, but most importantly, it increased the frequency of nonmosaic embryos and of transgene FISH signals, making it an interesting alternative for transgenic domestic animal production.

\section{Acknowledgments}

The authors are grateful to Dr. H. Ogino, Dr. E. Amaya, and Dr. J. Wittbrodt, for kindly providing the constructs ISceI-pBSII SK+ and pIS Pax6gfp. The authors also thank Dr. Elizabeth Crichton (bethiberg@aol.com) for her assistance in improving the English and to Dr. Gabriela Russo for her help with statistical analysis. The authors are also thank CIALE for donating semen.

\section{Appendix A. Supplementary data}

Supplementary data associated with this article can be found, in the online version, at http://dx.doi.org/10.1016/ j.theriogenology.2013.03.017.

\section{References}

[1] Gordon JW, Scangos GA, Plotkin DJ, Barbosa JA, Ruddle FH. Genetic transformation of mouse embryos by microinjection of purified DNA. Proc Natl Acad Sci U S A 1980;77:7380-4.

[2] Rossant J, Nutter L, Gertsenstein M. Engineering the embryo. Proc Natl Acad Sci U S A 2011;108:7659-60.

[3] Murakami M, Fahrudin M, Varisanga M, Suzuki T. Fluorescence expression by bovine embryos after pronuclear microinjection with the EGFP gene. J Vet Med Sci 1999;61:843-7.

[4] Cibelli JB, Stice SL, Golueke PJ, Kane JJ, Jerry J, Blackwell C, et al. Cloned transgenic calves produced from nonquiescent fetal fibroblasts. Science 1998;280:1256-8.

[5] Salamone D, Barañao L, Santos C, Bussmann L, Artuso J, Werning C, et al. High level expression of bioactive recombinant human growth hormone in the milk of a cloned transgenic cow. J Biotechnol 2006; 124:469-72.

[6] Richt JA, Kasinathan P, Hamir AN, Castilla J, Sathiyaseelan T, Vargas F, et al. Production of cattle lacking prion protein. Nat Biotechnol 2007;25:132-8.
[7] Meissner A, Jaenisch R. Mammalian nuclear transfer. Dev Dyn 2006; 235:2460-9.

[8] Thuan N, Kishigami S, Wakayama T. How to improve the success rate of mouse cloning technology. J Reprod Dev 2010;56:20-30.

[9] Ohtsuka M, Ogiwara S, Miura H, Mizutani A, Warita T, Sato M, et al. Pronuclear injection-based mouse targeted transgenesis for reproducible and highly efficient transgene expression. Nucleic Acids Res 2010;38:e198.

[10] Tasic B, Hippenmeyer S, Wang C, Gamboa M, Zong H, Chen-Tsai Y, et al. Site- specific integrase-mediated transgenesis in mice via pronuclear injection. Proc Natl Acad Sci U S A 2011;108:7902-7.

[11] Kim Y, Cha J. Chandrasegaran S. Hybrid restriction enzymes: zinc finger fusions to Fok I cleavage domain. Proc Natl Acad Sci U S A 1996;93:1156-60.

[12] Cermak T, Doyle E, Christian M, Wang L, Zhang Y, Schmidt C, et al. Efficient design and assembly of custom TALEN and other TAL effector-based constructs for DNA targeting. Nucleic Acids Res 2011; 39:e82.

[13] Bibikova M, Beumer K, Trautman J, Carroll D. Enhancing gene targeting with designed zinc finger nucleases. Science 2003;300:764.

[14] Ivics Z, Hackett PB, Plasterk RH, Izsvák Z. Molecular reconstruction of Sleeping Beauty, a Tc1-like transposon from fish, and its transposition in human cells. Cell 1997;91:501-10.

[15] Carlson DF, Geurts AM, Garbe JR, Park CW, Rangel-Filho A, O'Grady SM, et al. Efficient mammalian germline transgenesis by cis-enhanced Sleeping Beauty transposition. Transgenic Res 2011; 20:29-45.

[16] Garrels W, Mátés L, Holler S, Dalda A, Taylor U, Petersen B, et al. Germline transgenic pigs by Sleeping Beauty transposition in porcine zygotes and targeted integration in the pig genome. PLoS One 2011;6:e23573.

[17] Jakobsen JE, Li J, Kragh PM, Moldt B, Lin L, Liu Y, et al. Pig transgenesis by Sleeping Beauty DNA transposition. Transgenic Res 2011; 20:533-45.

[18] Macdonald J, Taylor L, Sherman A, Kawakami K, Takahashi Y, Sang HM, et al. Efficient genetic modification and germ-line transmission of primordial germ cells using piggyBac and Tol2 transposons. Proc Natl Acad Sci U S A 2012;109:E1466-72.

[19] Jacquier A, Dujon B. An intron-encoded protein is active in a gene conversion process that spreads an intron into a mitochondrial gene. Cell 1985;41:383-94.

[20] Thierry A, Dujon B. Nested chromosomal fragmentation in yeast using the meganuclease I-Scel: a new method for physical mapping of eukaryotic genomes. Nucleic Acids Res 1992;20:5625-31.

[21] Thermes V, Grabher C, Ristoratore F, Bourrat F, Choulika A, Wittbrodt J, et al. I- SceI meganuclease mediates highly efficient transgenesis in fish. Mech Dev 2002;118:91-8.

[22] Ogino H, McConnell WB, Grainger R. High-throughput transgenesis in Xenopus using I-SceI meganuclease. Nat Protoc 2006;1:1703-10.

[23] Nedelkovska H, Robert J. Optimized transgenesis in Xenopus laevis/ gilli isogenetic clones for immunological studies. Genesis 2011;50: 300-6.

[24] Casco-Robles M, Yamada S, Miura T, Chiba C. Simple and efficient transgenesis with I-Scel meganuclease in the newt, Cynops pyrrhogaster. Dev Dyn 2010;239:3275-84.

[25] Bevacqua RJ, Pereyra-Bonnet F, Olivera R, Hiriart MI, Sipowicz P, Fernandez-Martín R, et al. Production of IVF transgene-expressing bovine embryos using a novel strategy based on cell cycle inhibitors. Theriogenology 2012;78:57-68.

[26] Brinster RL, Chen HY, Trumbauer ME, Yagle MK, Palmiter RD. Factors affecting the efficiency of introducing foreign DNA into mice by microinjecting eggs. Proc Natl Acad Sci U S A 1985;82: 4438-42.

[27] Perry AC, Wakayama T, Kishikawa H, Kasai T, Okabe M, Toyoda Y, et al. Mammalian transgenesis by intracytoplasmic sperm injection. Science 1999;284:1180-3.

[28] Hartley KO, Hardcastle Z, Friday RV, Amaya E, Papalopulu N. Transgenic Xenopus embryos reveal that anterior neural development requires continued suppression of BMP signaling after gastrulation. Dev Biol 2001;238:168-84.

[29] Ikawa M, Kominami K, Yoshimura Y, Tanaka K, Nishimune Y, Okabe M. A rapid and non-invasive selection of transgenic embryos before implantation using green fluorescent protein (GFP). FEBS Lett 1995;375:125-8.

[30] Brackett BG, Oliphant G. Capacitation of rabbit spermatozoa in vitro. Biol Reprod 1975;12:260-74.

[31] Tervit HR, Whittingham DG, Rowson LE. Successful culture in vitro of sheep and cattle ova. J Reprod Fertil 1972;30:493-7.

[32] SAS Institute Inc. SAS/STAT. User's guide. Version 6. 4th ed., vol 1. Cary, NC: SAS Institute Inc; 1989. 
[33] Grabher C, Joly JS, Wittbrodt J. Highly efficient zebrafish transgenesis mediated by the meganuclease I-Scel. Methods Cell Biol 2004;77:381-401.

[34] Glaser T, Walton DS, Maas RL. Genomic structure, evolutionary conservation and aniridia mutations in the human PAX6 gene. Nat Genet 1992;2:232-9.

[35] Li HS, Yang J, Jacobson R, Pasko D, Sundin O. Pax-6 is first expressed in a region of ectoderm anterior to the early neural plate: implications for stepwise determination of the lens. Dev Biol 1994;162: 181-94.

[36] Mansouri A, Hallonet M, Gruss P. Pax genes and their roles in cell differentiation and development. Curr Opin Cell Biol 1996;8:851-7.

[37] Callaerts P, Halder G, Gehring WJ. PAX-6 in development and evolution. Ann Rev Neurosci 1997;20:483-532.

[38] Memili E, First NL. Control of gene expression at the onset of bovine embryonic development. Biol Reprod 1999;61:1198-207.

[39] Pan F, Chen Y, Loeber J, Henningfeld K, Pieler T. I-Scel meganuclease-mediated transgenesis in Xenopus. Dev Dyn 2006; 235:247-52.

[40] Pereyra-Bonnet F, Bevacqua R, La Rosa I, Sipowicz P, Radrizzani M, Fernandez-Martin R, et al. Novel methods to induce exogenous gene expression in SCNT, IVF and parthenogenic bovine embryos. Transgenic Res 2011;6:1379-88.

[41] Atef A, François P, Christian V, Marc-André S. The potential role of gap junction communication between cumulus cells and bovine oocytes during in vitro maturation. Mol Reprod Dev 2005;71: 358-67.

[42] Rosenblum CI, Chen H. In ovo transfection of chicken embryos using cationic liposomes. Transgenic Res 1995;4:192-8.

[43] Carballada R, Degefa T, Esponda P. Transfection of mouse eggs and embryos using DNA combined to cationic liposomes. Mol Reprod Dev 2000;56:360-5.

[44] Kroll KL, Amaya E. Transgenic Xenopus embryos from sperm nuclear transplantations reveal FGF signaling requirements during gastrulation. Development 1996;122:3173-83.

[45] Choulika A, Perrin A, Dujon B, Nicolas JF. Induction of homologous recombination in mammalian chromosomes by using the I-SceI system of Saccharomyces cerevisiae. Mol Cell Biol 1995;15: 1968-73.

[46] Iqbal K, Barg-Kues B, Broll S, Bode J, Niemann H, Kues W. Cytoplasmic injection of circular plasmids allows targeted expression in mammalian embryos. Biotechniques 2009;47:959-68.

[47] Etkin LD, Pearman B. Distribution, expression and germ line transmission of exogenous DNA sequences following microinjection into Xenopus laevis eggs. Development 1987;99:15-23.

[48] Etkin L, Pearman B, Ansah-Yiadom R. Replication of injected DNA templates in Xenopus embryos. Exp Cell Res 1987;169: 468-77.

[49] Garrick D, Fiering S, Martin DI, Whitelaw E. Repeat-induced gene silencing in mammals. Nat Genet 1998;18:56-9. 


\section{Supplementary FISH images}

Blastomere signals were evaluated using direct microscopic visualization. In addition, images of the blastomere signals were recorded with an Optronics camera. However, in many cases, fluorescence fading took place after prolonged exposure, and therefore, the fluorescence detected in the pictures was not as strong as the one observed using direct visualization immediately after exposure. In this Supplement, we include images of 30 of the blastomeres evaluated for this study.

Each figure in this Supplementary material is organized as panel (A), panel (B), and coupled image (panel
C); (A) corresponds to the exact picture taken using the microscope after the FISH procedure (the images were cropped for better presentation); (B) corresponds to the same pictures after background removal using the contrast enhancement tool of Image Pro Plus. The final image $(\mathrm{C})$ with the red and green signals coupled to the nuclei, was generated with the image operations tool of Image Pro Plus or with similar tools from Photoshop by superposition of the images shown in (B).

The first picture set corresponds to FISH on I-Scel transgenesis embryos.
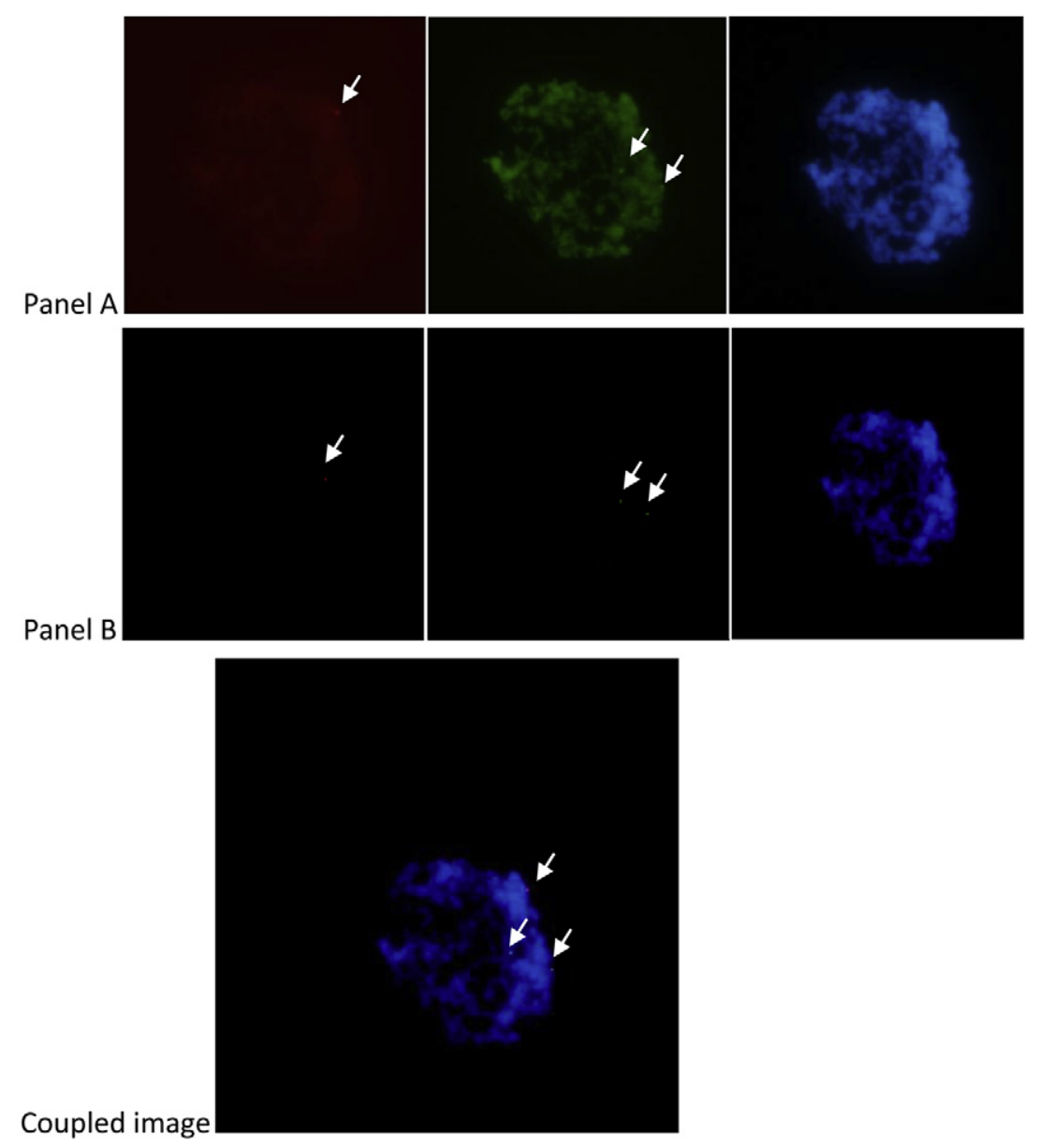

Supplementary Fig. 1. Blastomere with one transgene and two control signals. (A) Microscopic images after the FISH procedure. (B) Same images as in (A) after background removal. In (A) and (B), the left panel shows a gfp rhodamine-labeled probe (red signal); the middle panel, a bovine chromosome 19 pericentromeric probe labeled with FITC (green signal); and right panel, DAPI staining of chromosomes. (C) Image with the red and green signals coupled to the nuclei, generated with the image operations tool of Image Pro Plus or with similar tools from Photoshop, by superposition of the images shown in (B). 


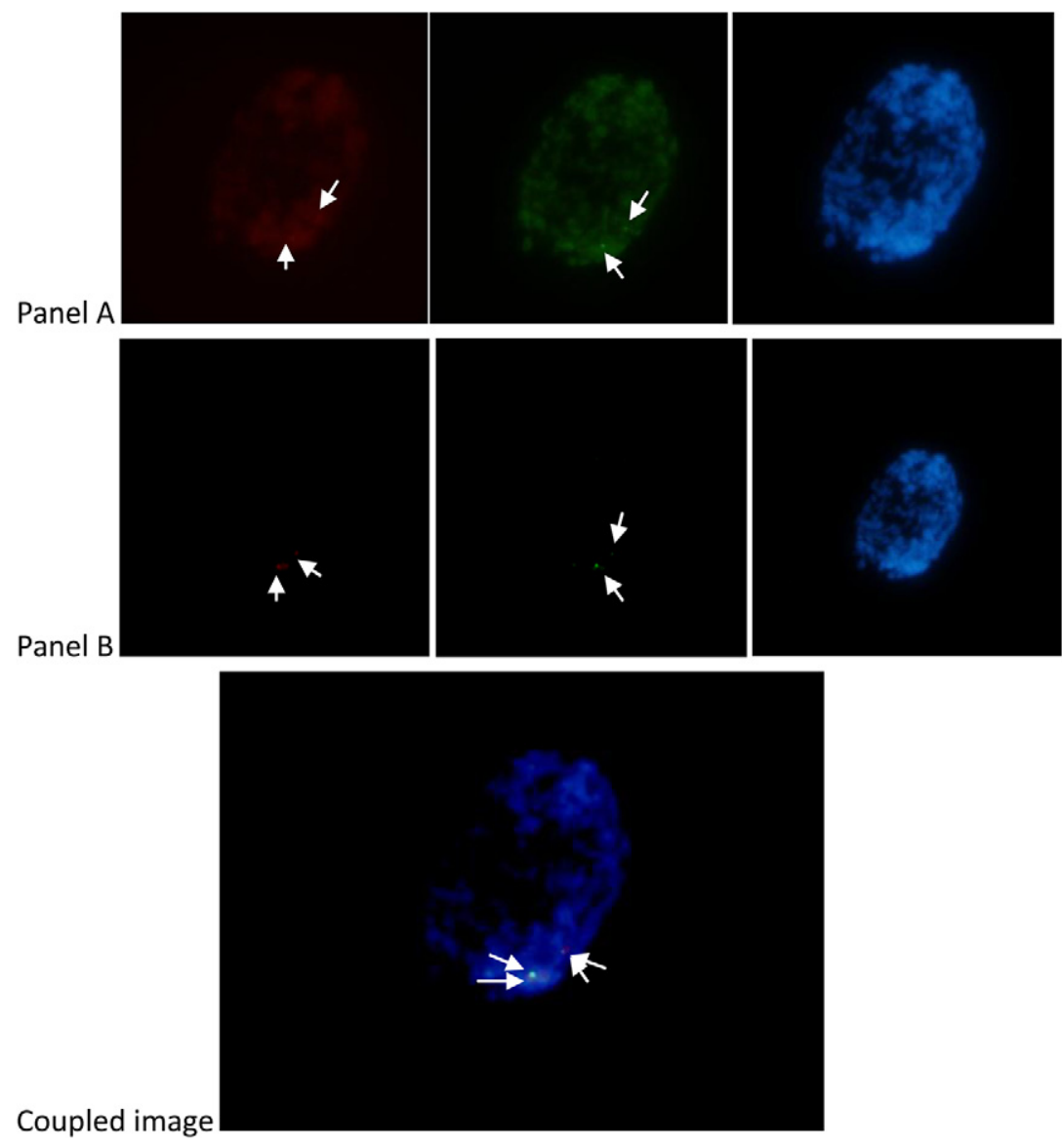

Supplementary Fig. 2. Blastomere with two transgene signals (not as clear in the picture as after direct microscopic visualization) and two control signals. (A) Microscopic images after the FISH procedure. (B) Same images as in (A) after background removal. In (A) and (B), the left panel shows a gfp rhodamine-labeled probe (red signal); the middle panel, a bovine chromosome 19 pericentromeric probe labeled with FITC (green signal); and right panel, DAPI staining of chromosomes. (C) Image with the red and green signals coupled to the nuclei, generated with the image operations tool of Image Pro Plus or with similar tools from Photoshop, by superposition of the images shown in (B). 


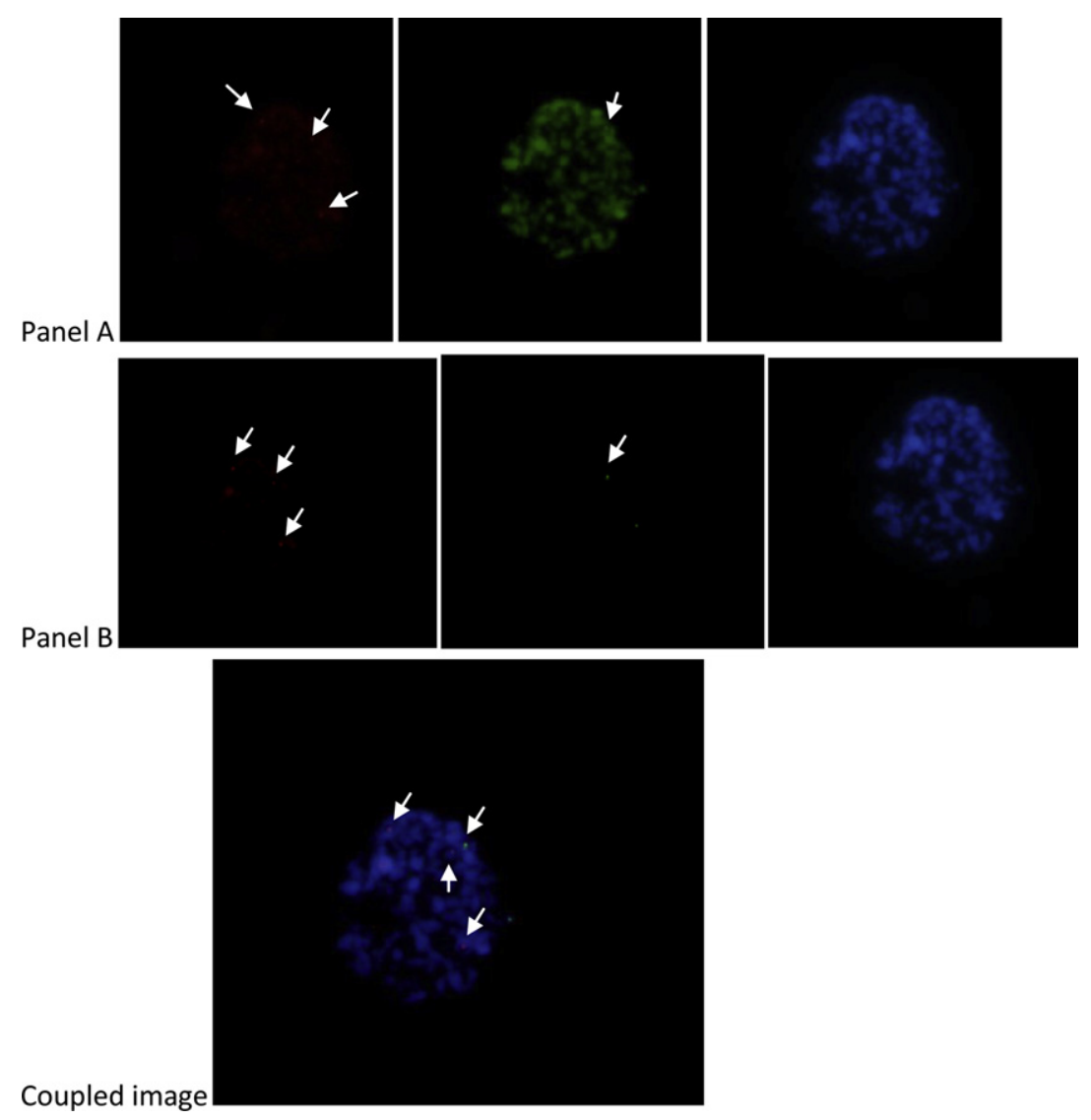

Supplementary Fig. 3. Blastomere with three transgene signals and only one chromosome signal (not well appreciated in the picture). (A) Microscopic images after the FISH procedure. (B) Same images as in (A) after background removal. In (A) and (B), the left panel shows a gfp rhodamine-labeled probe (red signal); the middle panel, a bovine chromosome 19 pericentromeric probe labeled with FITC (green signal); and right panel, DAPI staining of chromosomes. (C) Image with the red and green signals coupled to the nuclei, generated with the image operations tool of Image Pro Plus or with similar tools from Photoshop, by superposition of the images shown in (B). 


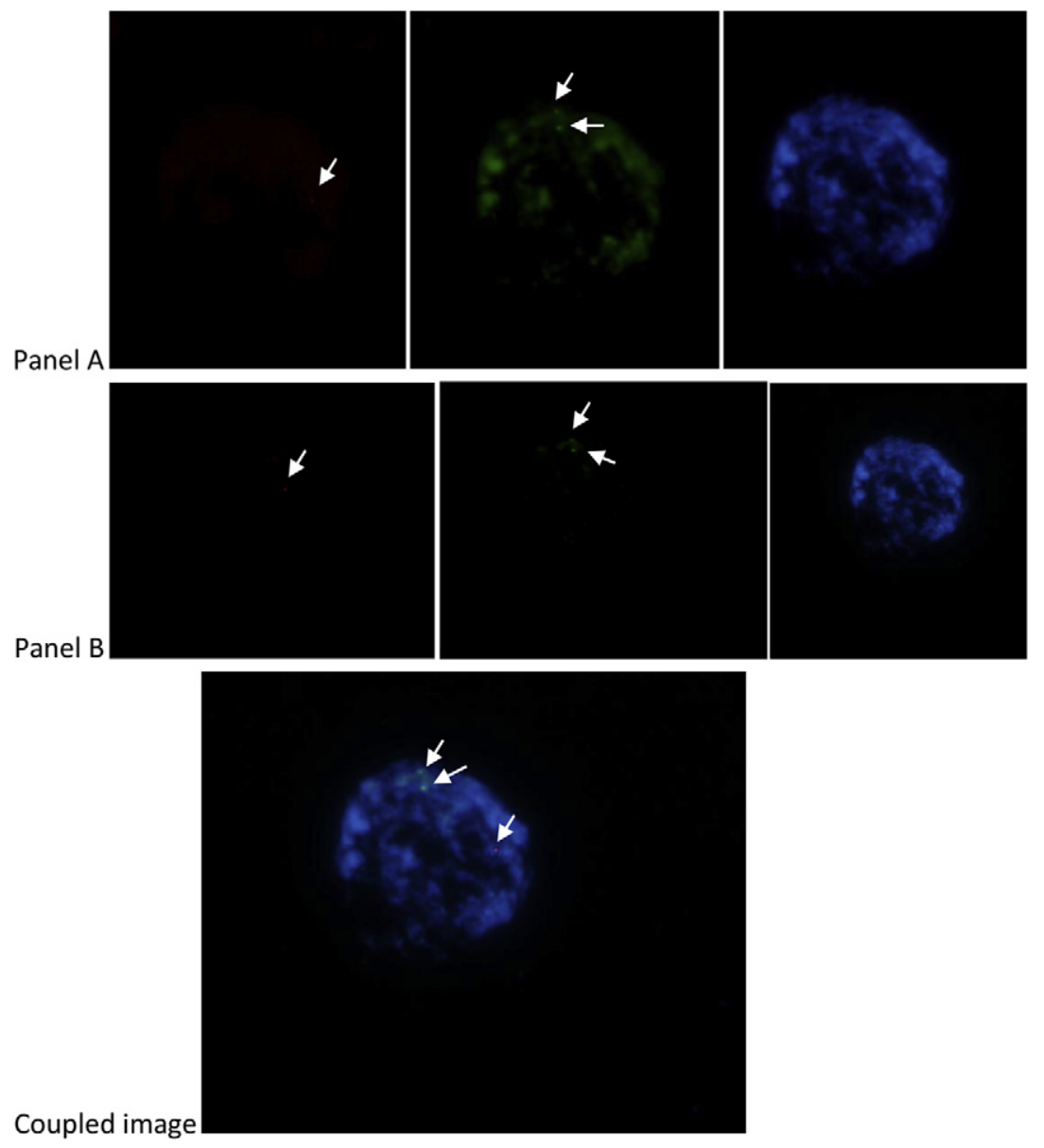

Supplementary Fig. 4. Blastomere with one transgene and two control signals. (A) Microscopic images after the FISH procedure. (B) Same images as in (A) after background removal. In (A) and (B), the left panel shows a $g f p$ rhodamine-labeled probe (red signal); the middle panel, a bovine chromosome 19 pericentromeric probe labeled with FITC (green signal); and right panel, DAPI staining of chromosomes. (C) Image with the red and green signals coupled to the nuclei, generated with the image operations tool of Image Pro Plus or with similar tools from Photoshop, by superposition of the images shown in (B). 

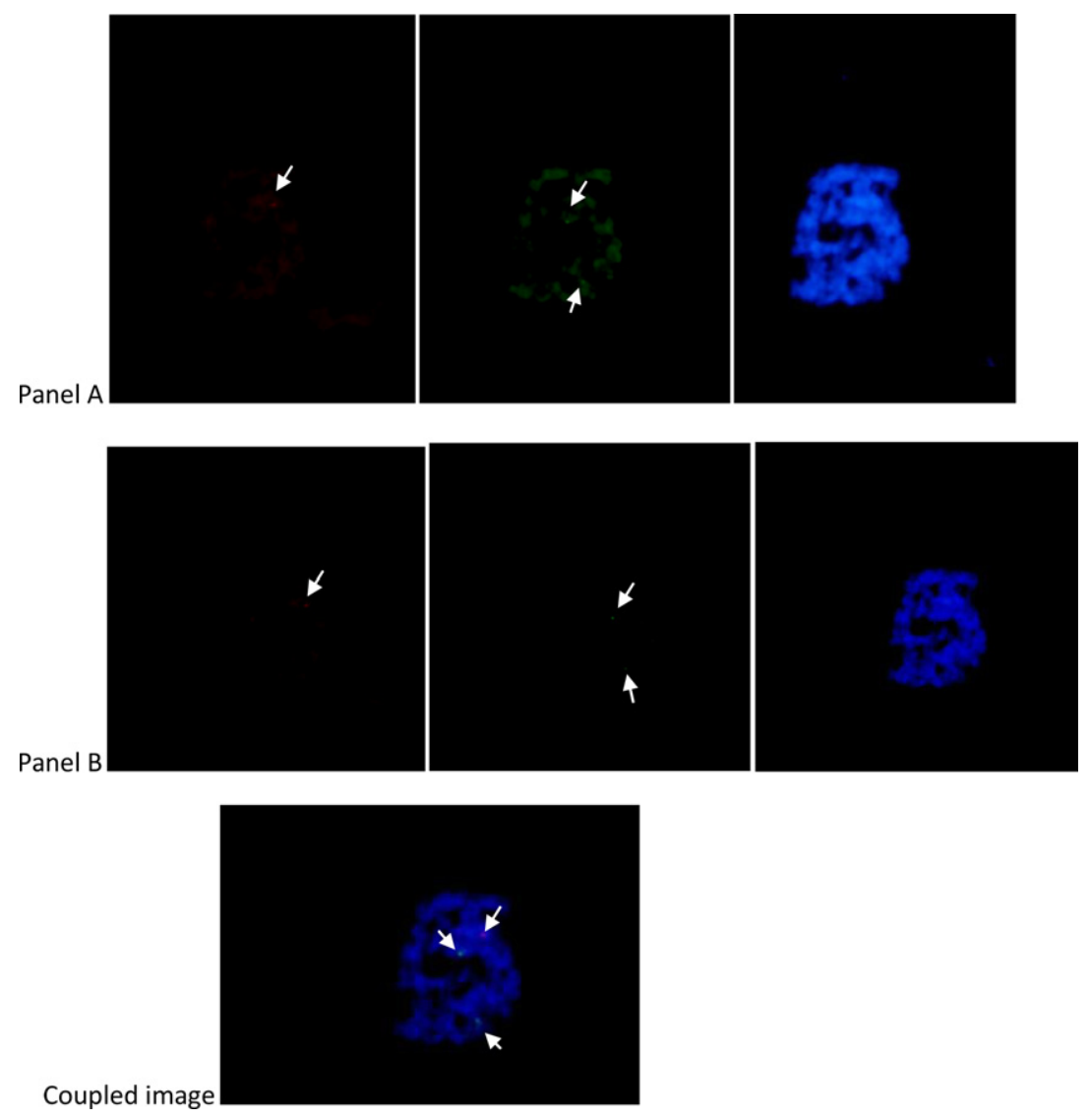

Supplementary Fig. 5. Blastomere with one transgene and two control signals. (A) Microscopic images after the FISH procedure. (B) Same images as in (A) after background removal. In (A) and (B), the left panel shows a $g f p$ rhodamine-labeled probe (red signal); the middle panel, a bovine chromosome 19 pericentromeric probe labeled with FITC (green signal); and right panel, DAPI staining of chromosomes. (C) Image with the red and green signals coupled to the nuclei, generated with the image operations tool of Image Pro Plus or with similar tools from Photoshop, by superposition of the images shown in (B). 

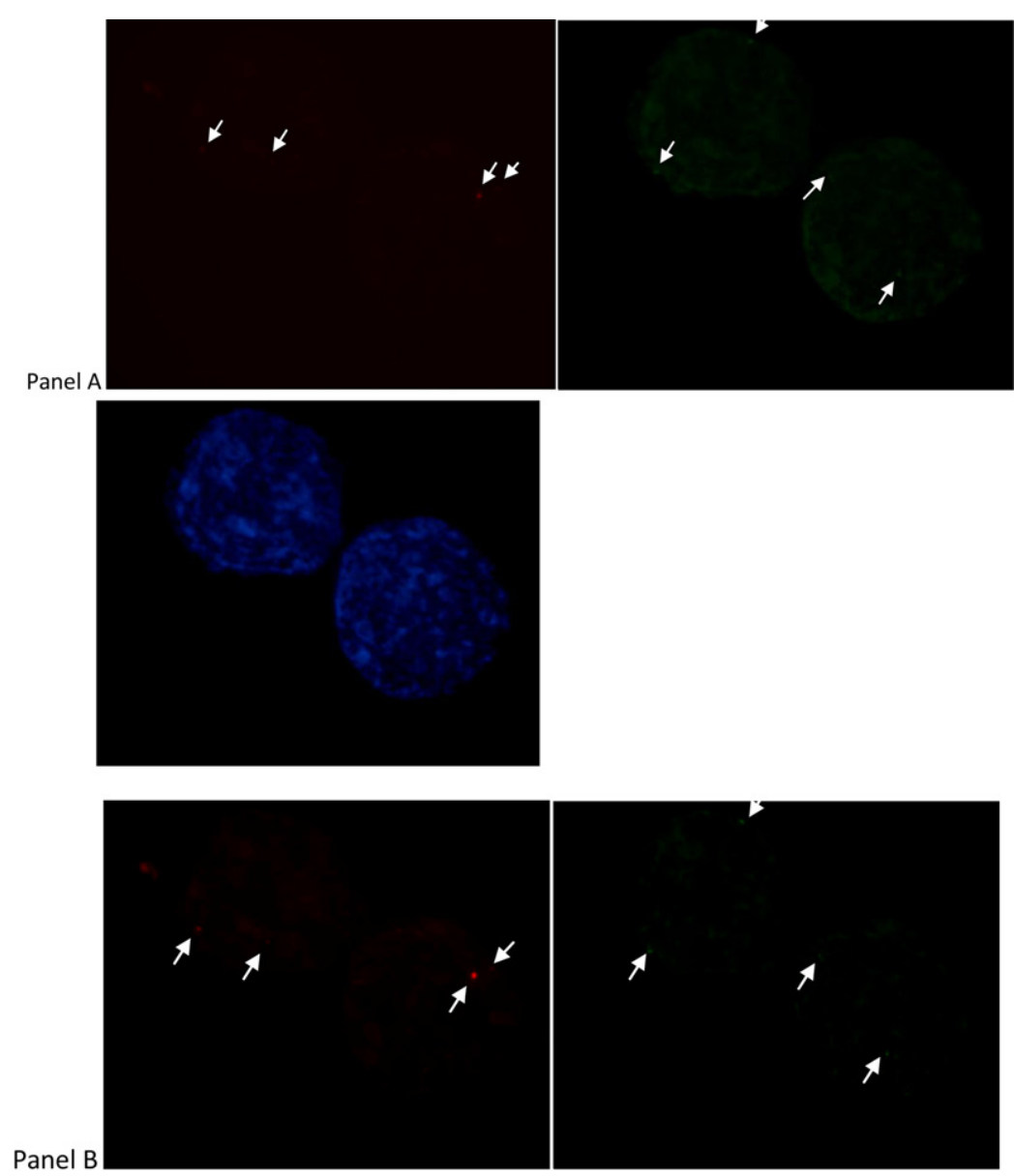

Panel B
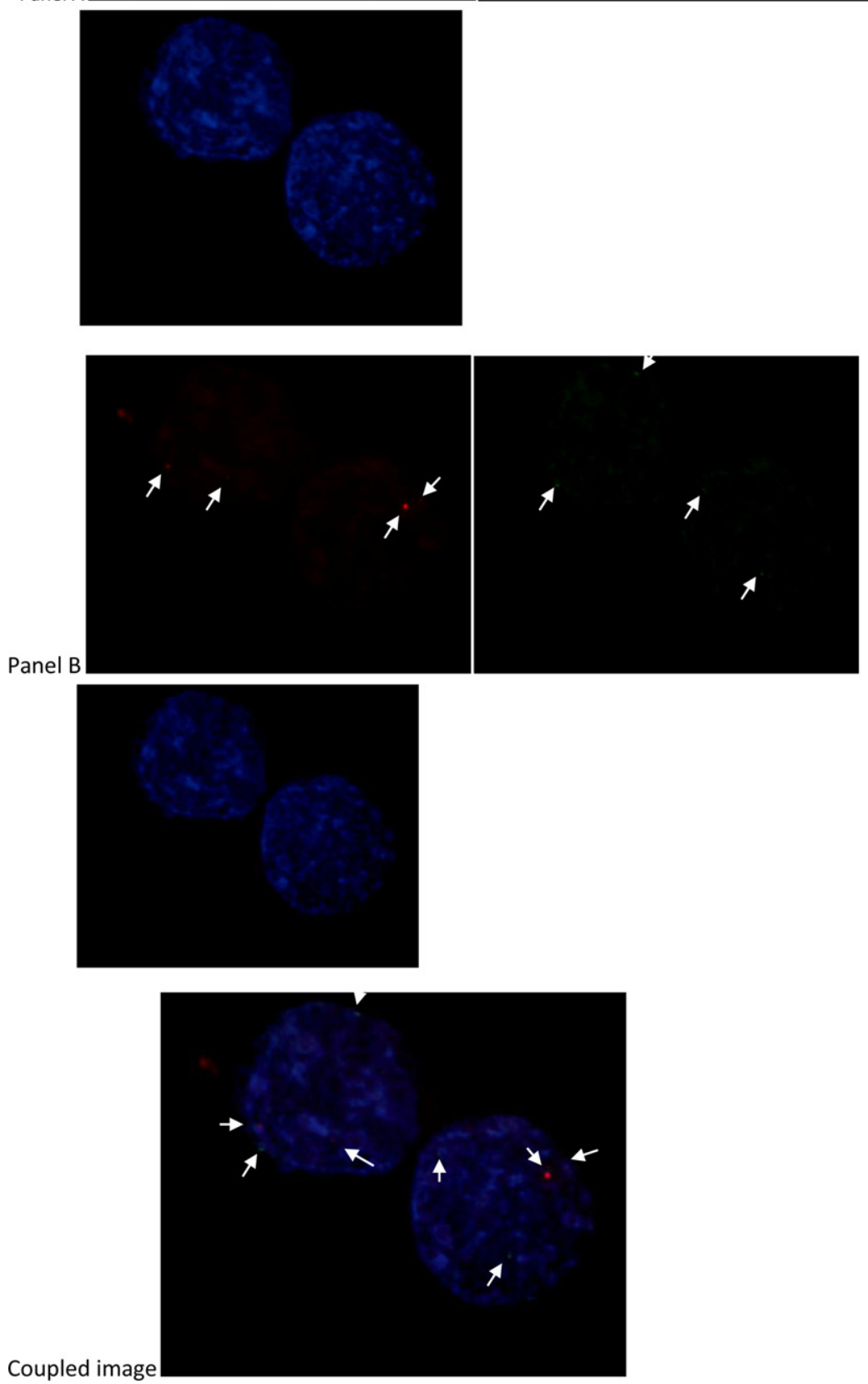

Supplementary Fig. 6. Two blastomeres, both of them with two transgene and two control signals. (A) Microscopic images after the FISH procedure. (B) Same images as in (A) after background removal. In (A) and (B), the left panel shows a $g f p$ rhodamine-labeled probe (red signal); the middle panel, a bovine chromosome 19 pericentromeric probe labeled with FITC (green signal); and right panel, DAPI staining of chromosomes. (C) Image with the red and green signals coupled to the nuclei, generated with the image operations tool of Image Pro Plus or with similar tools from Photoshop, by superposition of the images shown in (B). 

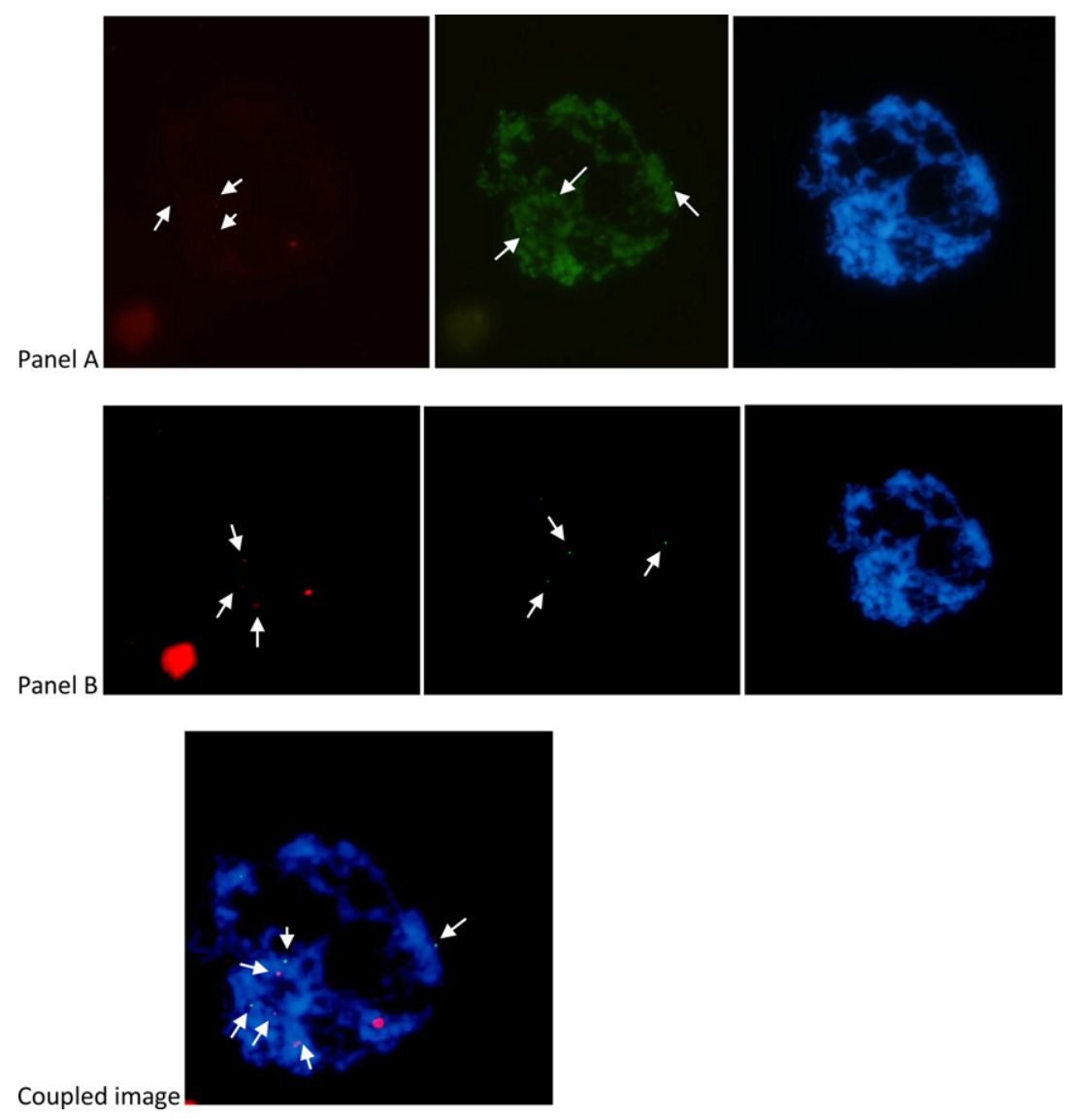

Supplementary Fig. 7. Blastomere with a trisomy (three control signals) and also three transgene signals (not showing a very strong fluorescence in the picture, because of the presence of some strong unspecific fluorescence). (A) Microscopic images after the FISH procedure. (B) Same images as in (A) after background removal. In (A) and (B), the left panel shows a gfp rhodamine-labeled probe (red signal); the middle panel, a bovine chromosome 19 pericentromeric probe labeled with FITC (green signal); and right panel, DAPI staining of chromosomes. (C) Image with the red and green signals coupled to the nuclei, generated with the image operations tool of Image Pro Plus or with similar tools from Photoshop, by superposition of the images shown in (B). 


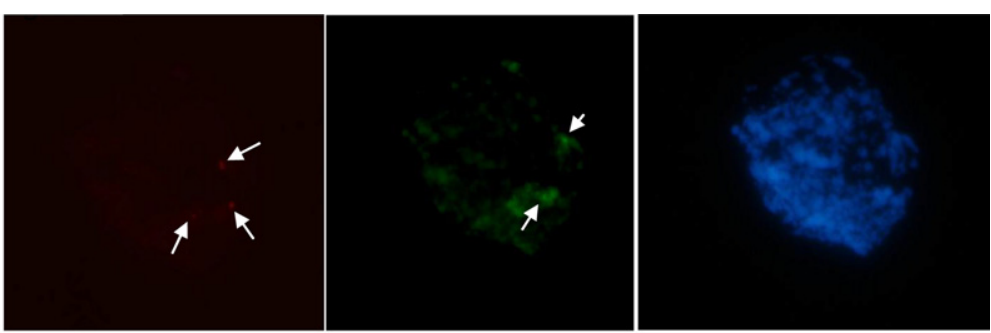

Panel A
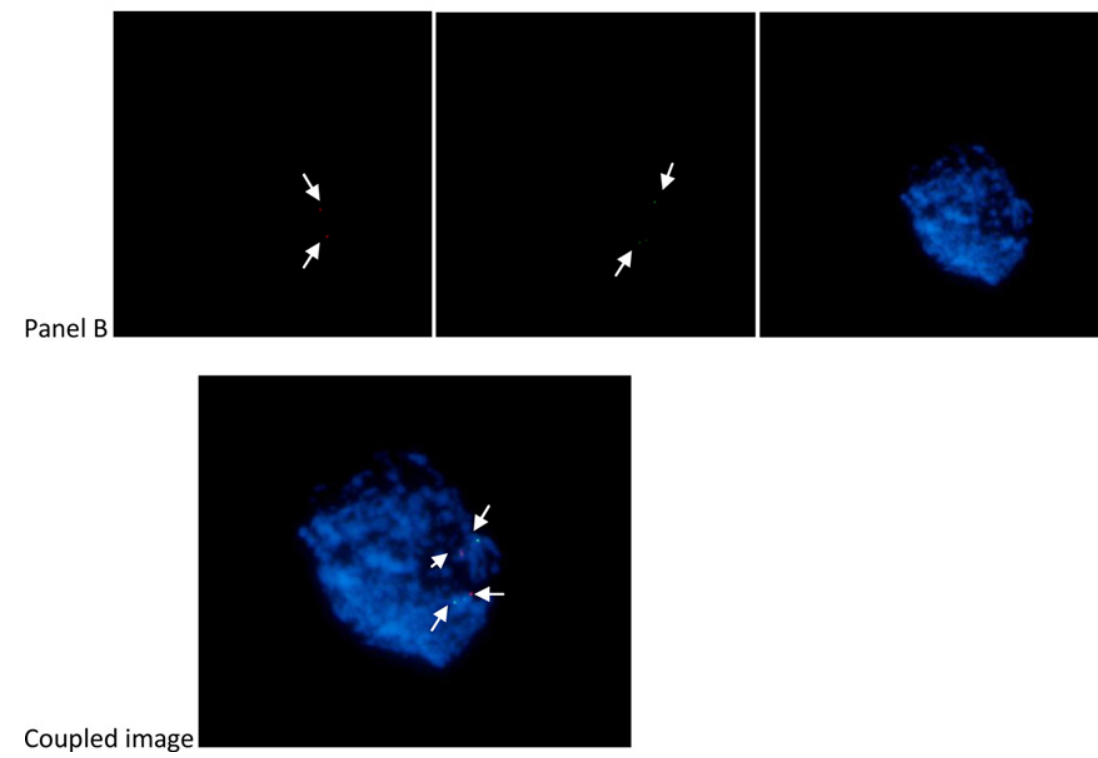

Supplementary Fig. 8. Blastomere with three transgene signals, one of them lost in (B) after increase of contrast to allow visualization of signals in the coupled image, and two control signals. (A) Microscopic images after the FISH procedure. (B) Same images as in (A) after background removal. In (A) and (B), the left panel shows a gfp rhodamine-labeled probe (red signal); the middle panel, a bovine chromosome 19 pericentromeric probe labeled with FITC (green signal); and right panel, DAPI staining of chromosomes. (C) Image with the red and green signals coupled to the nuclei, generated with the image operations tool of Image Pro Plus or with similar tools from Photoshop, by superposition of the images shown in (B). 


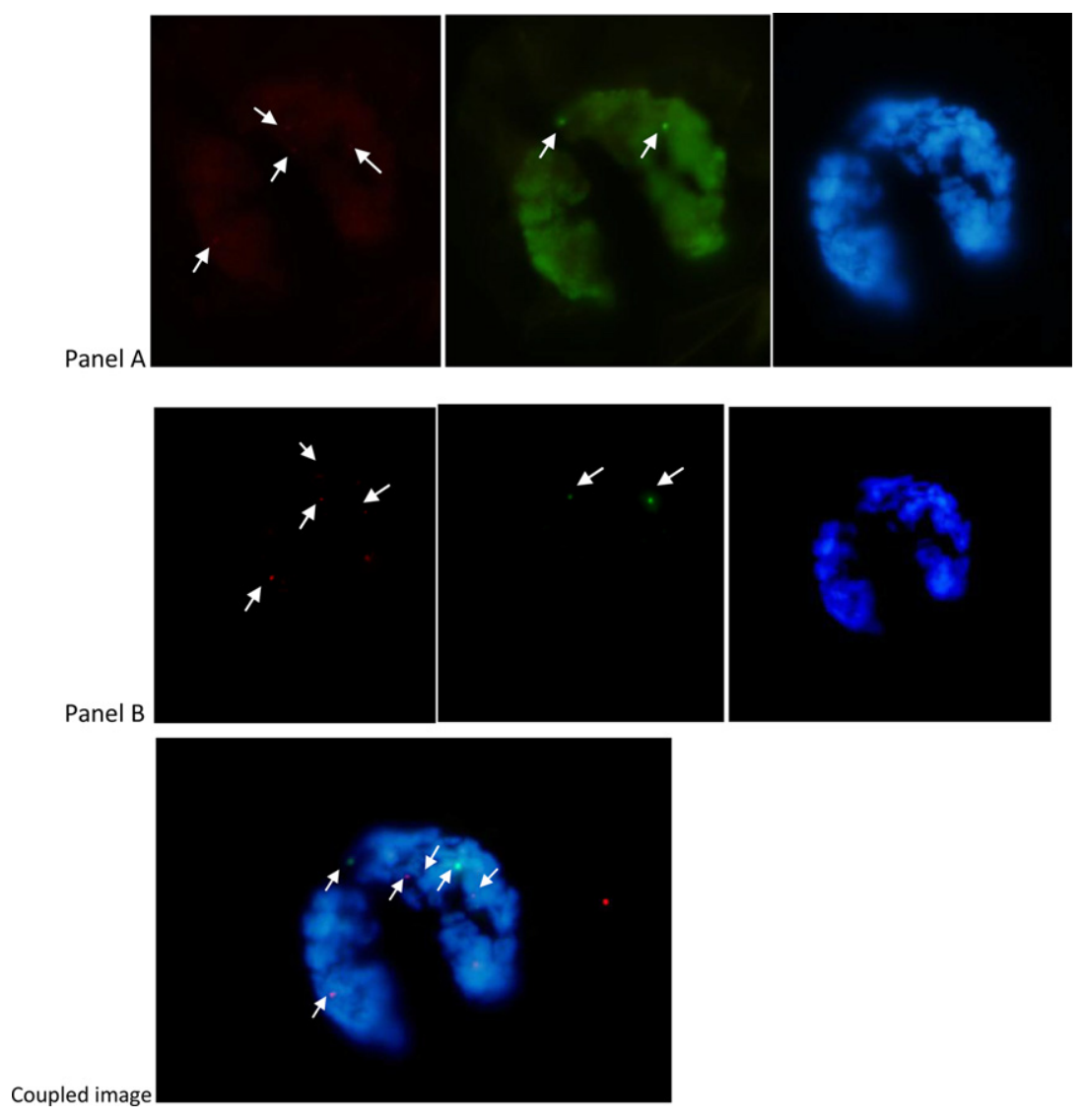

Supplementary Fig. 9. Blastomere with four transgene and two control signals. (A) Microscopic images after the FISH procedure. (B) Same images as in (A) after background removal. In (A) and (B), the left panel shows a $g f p$ rhodamine-labeled probe (red signal); the middle panel, a bovine chromosome 19 pericentromeric probe labeled with FITC (green signal); and right panel, DAPI staining of chromosomes. (C) Image with the red and green signals coupled to the nuclei, generated with the image operations tool of Image Pro Plus or with similar tools from Photoshop, by superposition of the images shown in (B). 


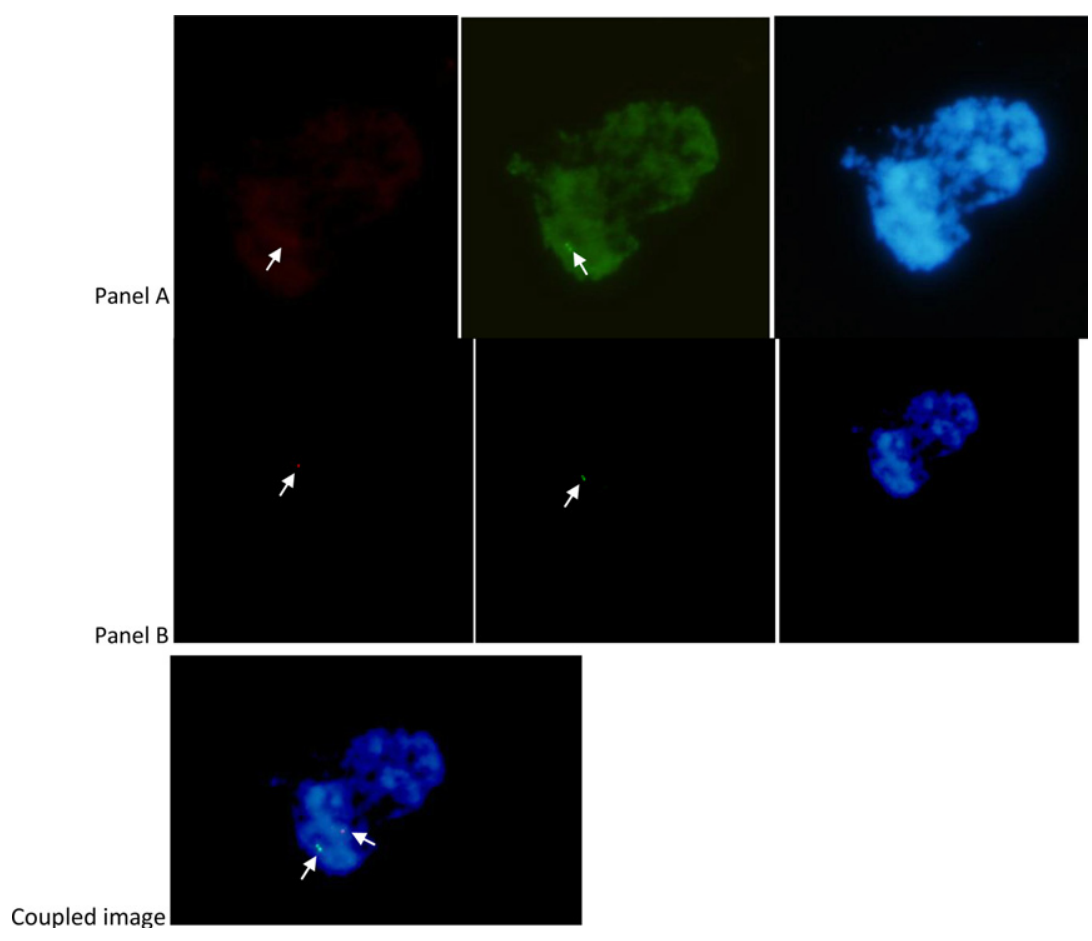

Supplementary Fig. 10. Blastomere with one transgene, more clearly seen in (A) than in (B), and one control signal, in pairs, possibly related to each sister chromatide. (A) Microscopic images after the FISH procedure. (B) Same images as in (A) after background removal. In (A) and (B), the left panel shows a gfp rhodamine-labeled probe (red signal); the middle panel, a bovine chromosome 19 pericentromeric probe labeled with FITC (green signal); and right panel, DAPI staining of chromosomes. (C) Image with the red and green signals coupled to the nuclei, generated with the image operations tool of Image Pro Plus or with similar tools from Photoshop, by superposition of the images shown in (B). 

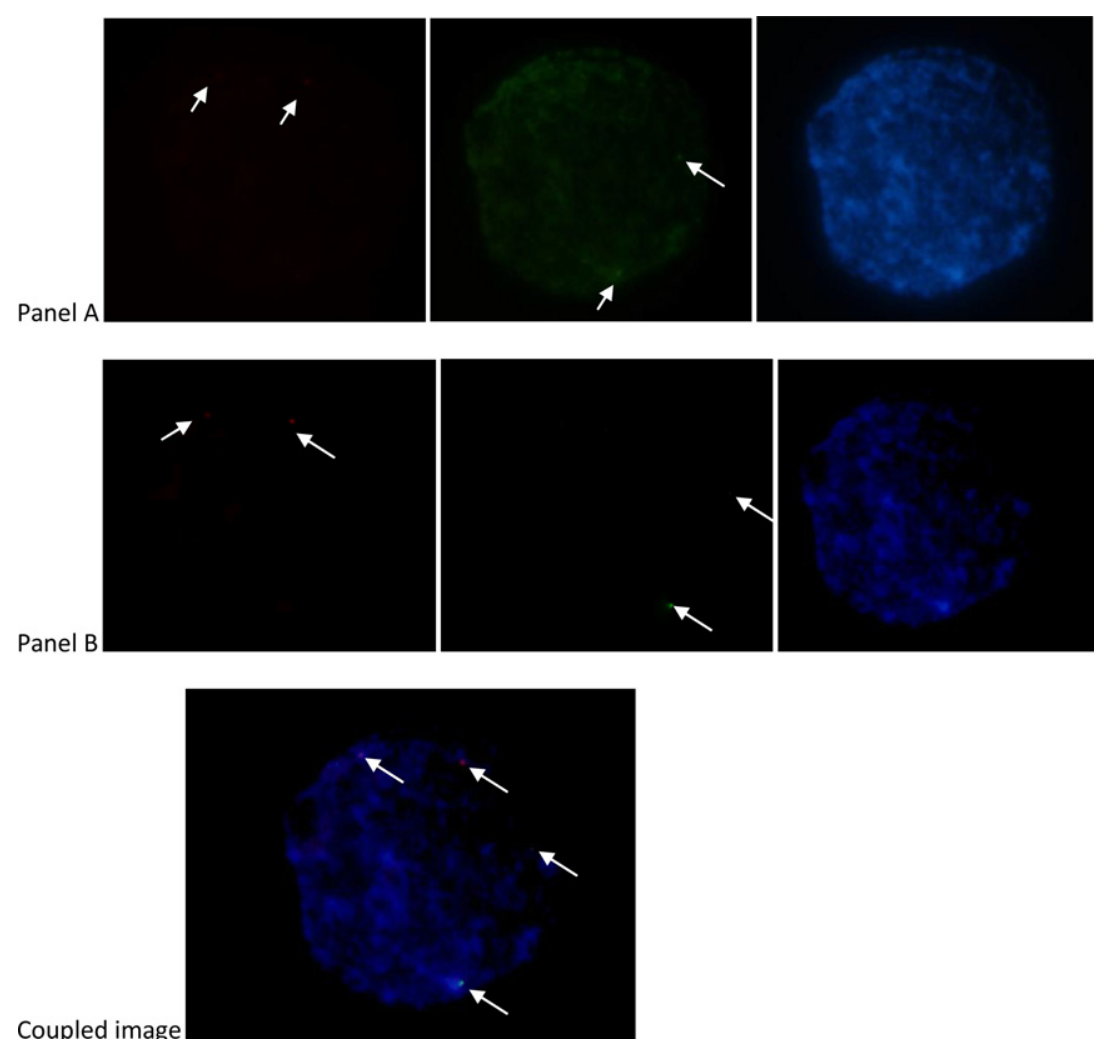

Supplementary Fig. 11. Blastomere with two transgene and two control signals. (A) Microscopic images after the FISH procedure. (B) Same images as in (A) after background removal. In (A) and (B), the left panel shows a $g f p$ rhodamine-labeled probe (red signal); the middle panel, a bovine chromosome 19 pericentromeric probe labeled with FITC (green signal); and right panel, DAPI staining of chromosomes. (C) Image with the red and green signals coupled to the nuclei, generated with the image operations tool of Image Pro Plus or with similar tools from Photoshop, by superposition of the images shown in (B). 

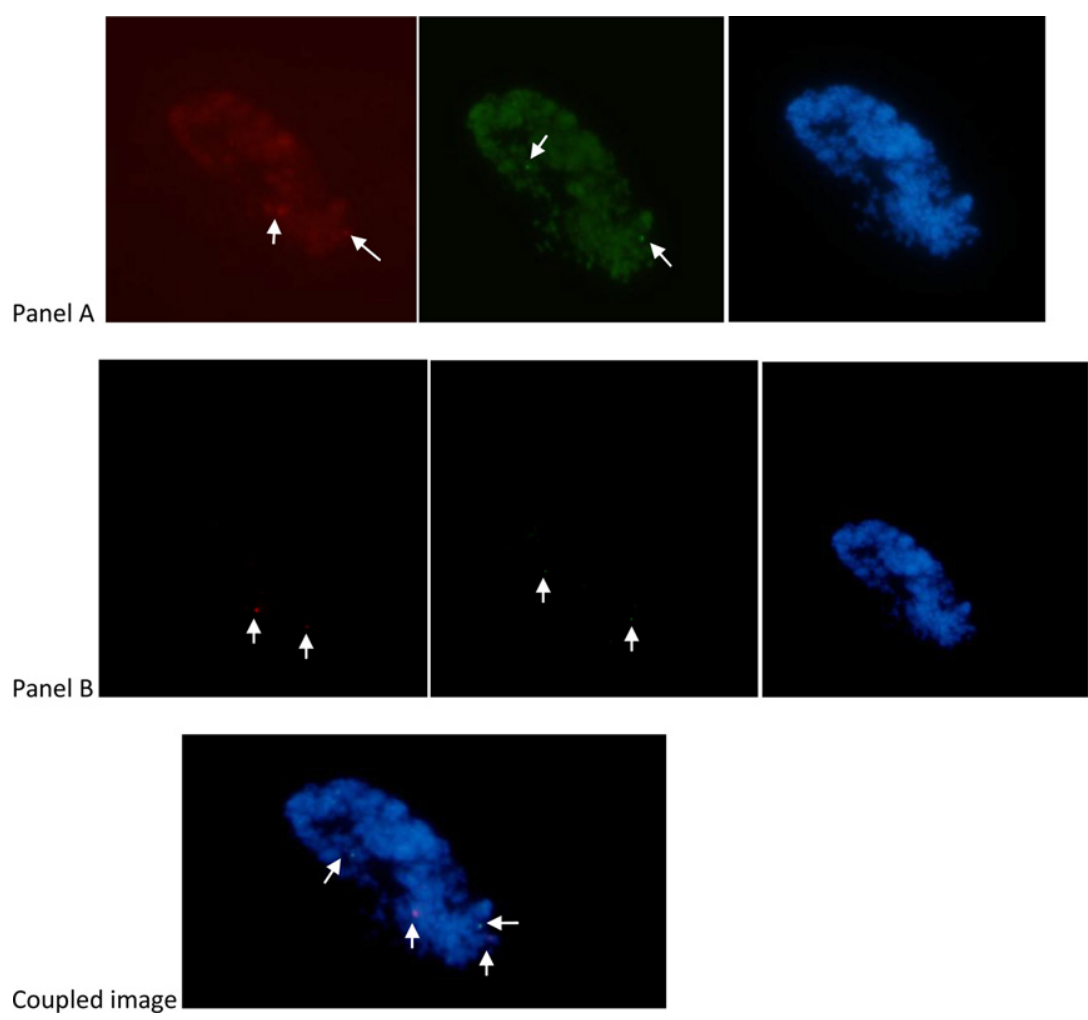

Supplementary Fig. 12. Blastomere with two transgene and two control signals. (A) Microscopic images after the FISH procedure. (B) Same images as in (A) after background removal. In (A) and (B), the left panel shows a gfp rhodamine-labeled probe (red signal); the middle panel, a bovine chromosome 19 pericentromeric probe labeled with FITC (green signal); and right panel, DAPI staining of chromosomes. (C) Image with the red and green signals coupled to the nuclei, generated with the image operations tool of Image Pro Plus or with similar tools from Photoshop, by superposition of the images shown in (B). 


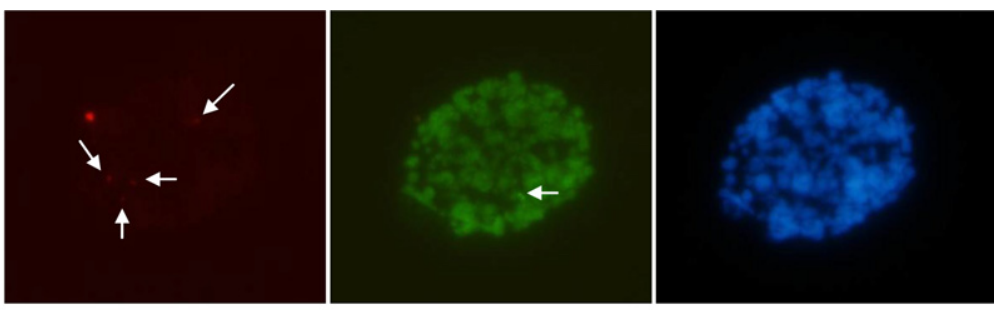

Panel A
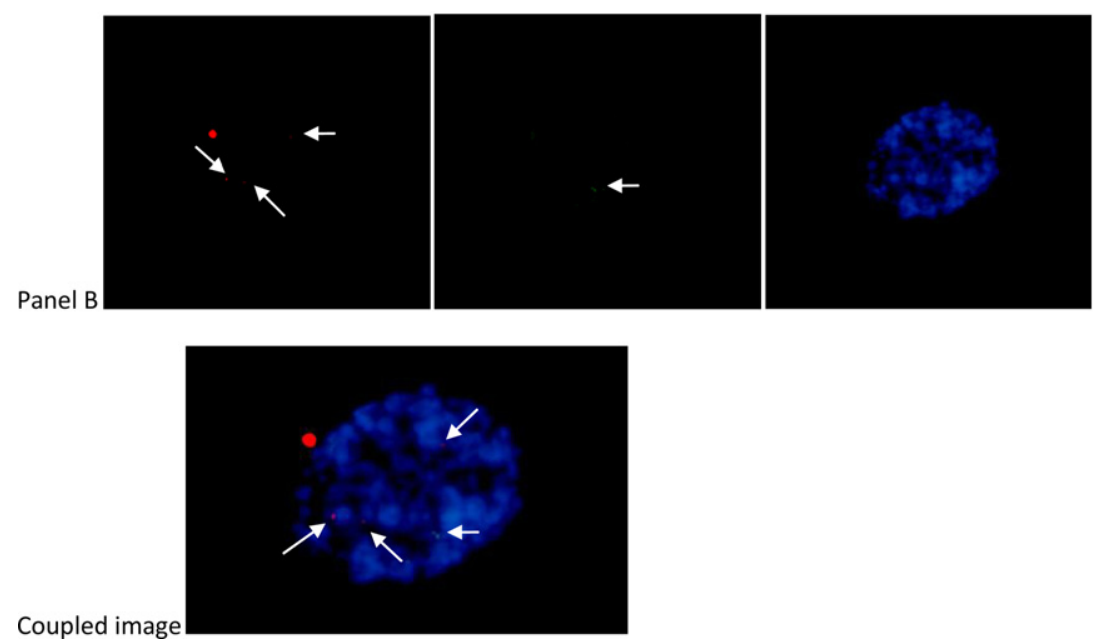

Supplementary Fig. 13. Blastomere with four transgene signals (one too weak to persist after background removal) and one control signal (in pairs, not very clear in this picture). (A) Microscopic images after the FISH procedure. (B) Same images as in (A) after background removal. In (A) and (B), the left panel shows a gfp rhodamine-labeled probe (red signal); the middle panel, a bovine chromosome 19 pericentromeric probe labeled with FITC (green signal); and right panel, DAPI staining of chromosomes. (C) Image with the red and green signals coupled to the nuclei, generated with the image operations tool of Image Pro Plus or with similar tools from Photoshop, by superposition of the images shown in (B). 


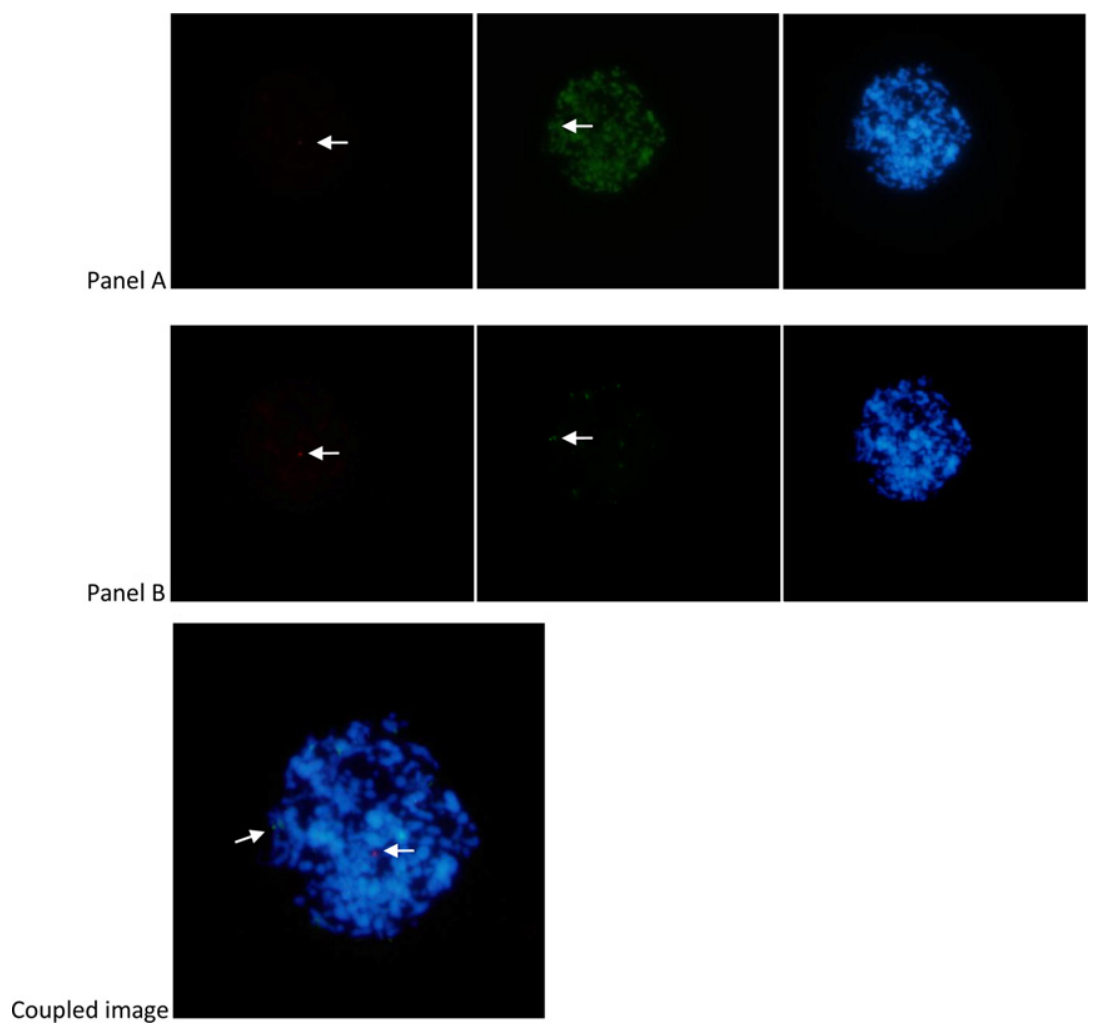

Supplementary Fig. 14. Blastomere with one transgene and one control signal (not clear in the picture because of the background). (A) Microscopic images after the FISH procedure. (B) Same images as in (A) after background removal. In (A) and (B), the left panel shows a gfp rhodamine-labeled probe (red signal); the middle panel, a bovine chromosome 19 pericentromeric probe labeled with FITC (green signal); and right panel, DAPI staining of chromosomes. (C) Image with the red and green signals coupled to the nuclei, generated with the image operations tool of Image Pro Plus or with similar tools from Photoshop, by superposition of the images shown in (B). 

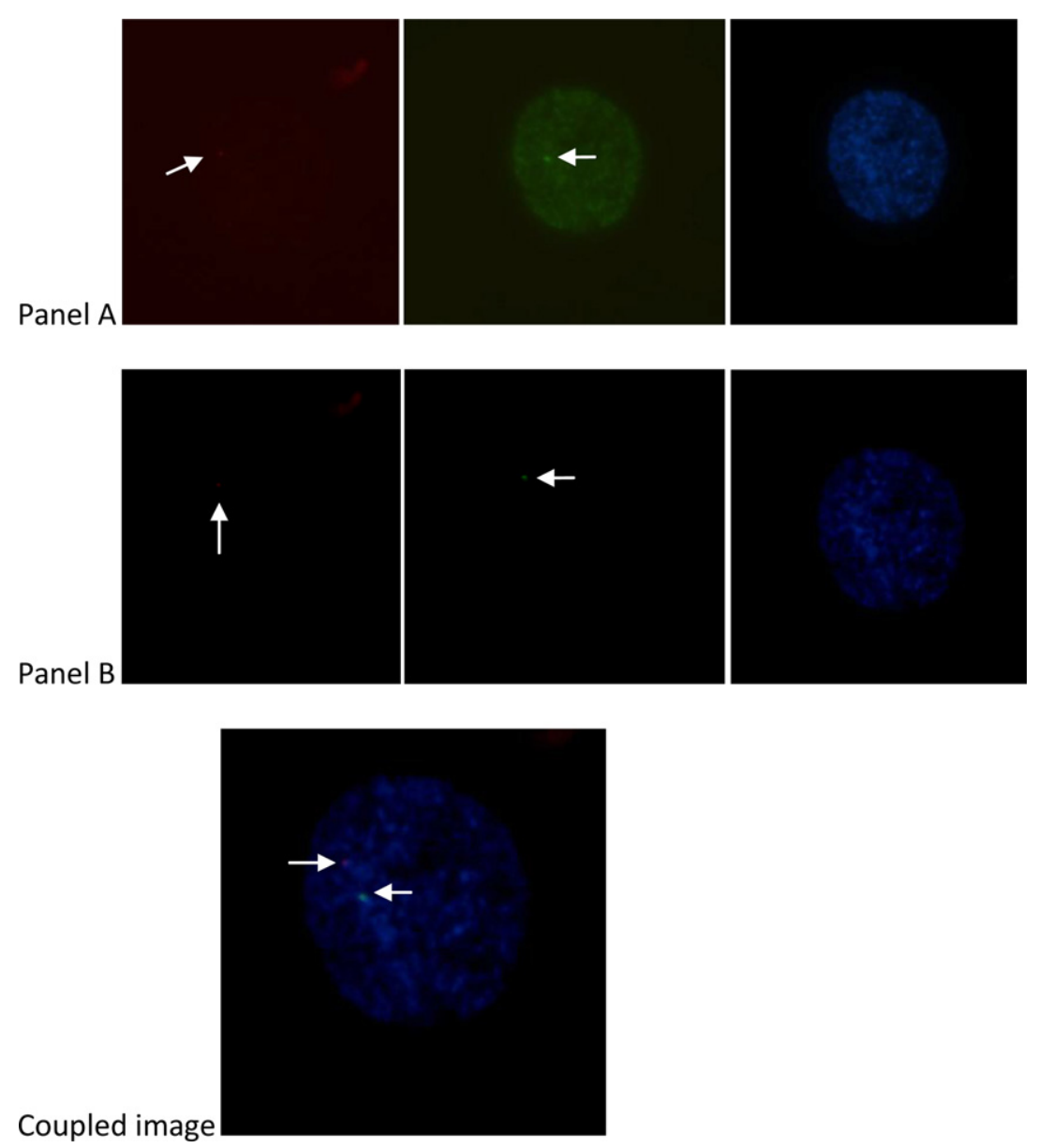

Supplementary Fig. 15. Blastomere with one transgene and one control signal. (A) Microscopic images after the FISH procedure. (B) Same images as in (A) after background removal. In (A) and (B), the left panel shows a gfp rhodamine-labeled probe (red signal); the middle panel, a bovine chromosome 19 pericentromeric probe labeled with FITC (green signal); and right panel, DAPI staining of chromosomes. (C) Image with the red and green signals coupled to the nuclei, generated with the image operations tool of Image Pro Plus or with similar tools from Photoshop, by superposition of the images shown in (B). 


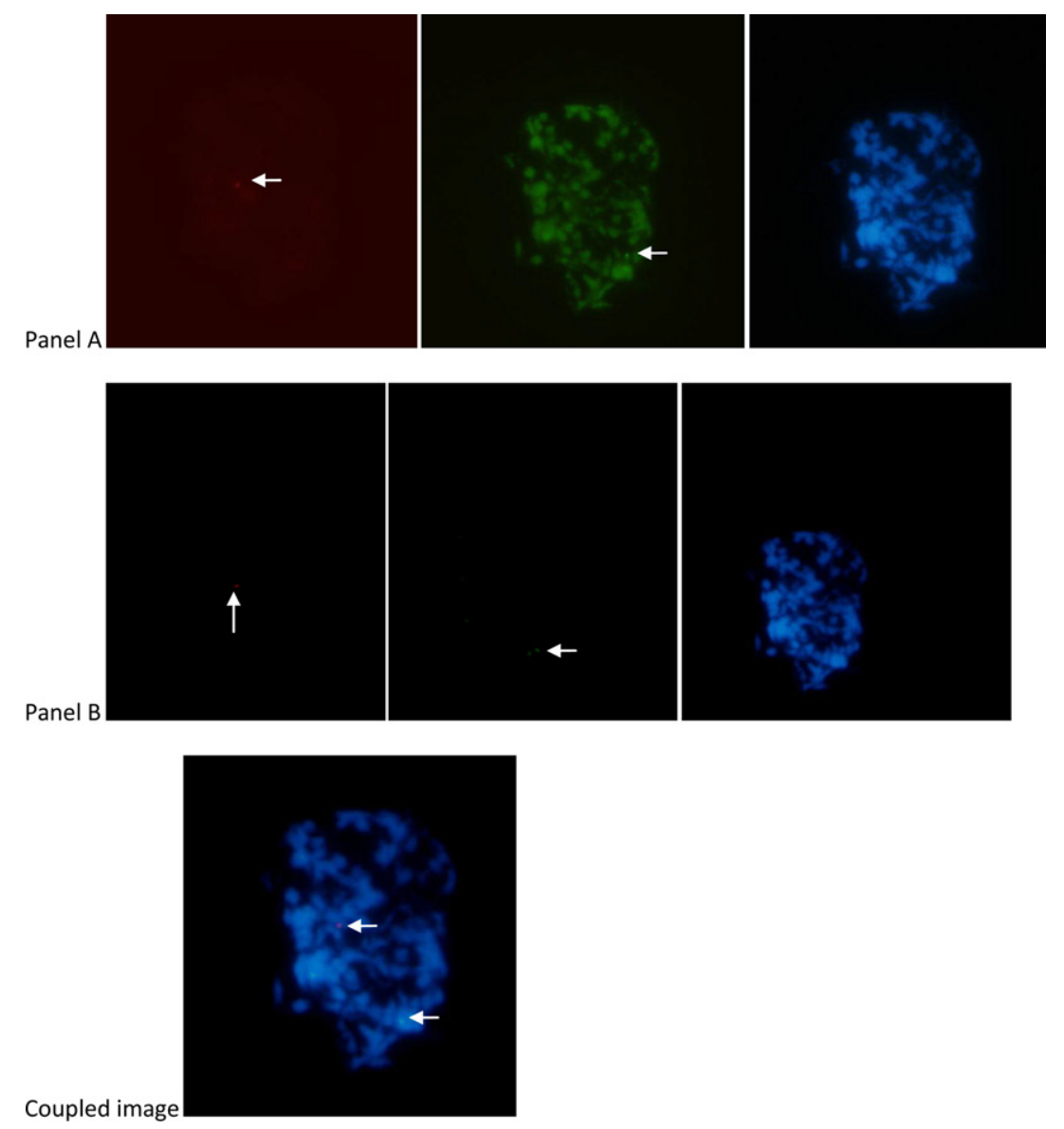

Supplementary Fig. 16. Blastomere with one transgene and one control signal. (A) Microscopic images after the FISH procedure. (B) Same images as in (A) after background removal. In (A) and (B), the left panel shows a gfp rhodamine-labeled probe (red signal); the middle panel, a bovine chromosome 19 pericentromeric probe labeled with FITC (green signal); and right panel, DAPI staining of chromosomes. (C) Image with the red and green signals coupled to the nuclei, generated with the image operations tool of Image Pro Plus or with similar tools from Photoshop, by superposition of the images shown in (B). 

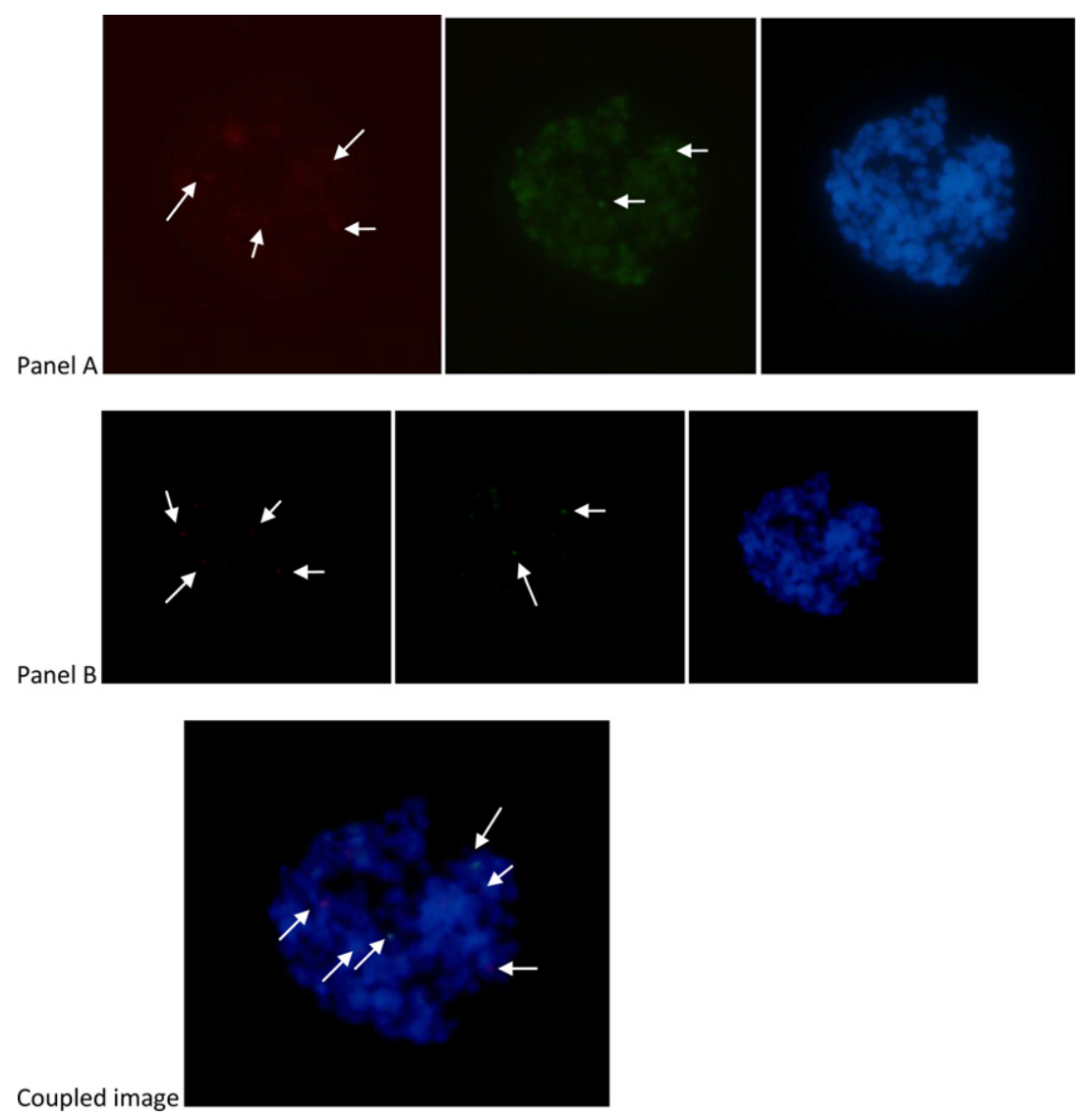

Supplementary Fig. 17. Blastomere with four transgene and two control signals. (A) Microscopic images after the FISH procedure. (B) Same images as in (A) after background removal. In (A) and (B), the left panel shows a gfp rhodamine-labeled probe (red signal); the middle panel, a bovine chromosome 19 pericentromeric probe labeled with FITC (green signal); and right panel, DAPI staining of chromosomes. (C) Image with the red and green signals coupled to the nuclei, generated with the image operations tool of Image Pro Plus or with similar tools from Photoshop, by superposition of the images shown in (B). 
The second group of pictures correspond to FISH performed on transgene-alone transgenesis-derived embryos.

Panel A

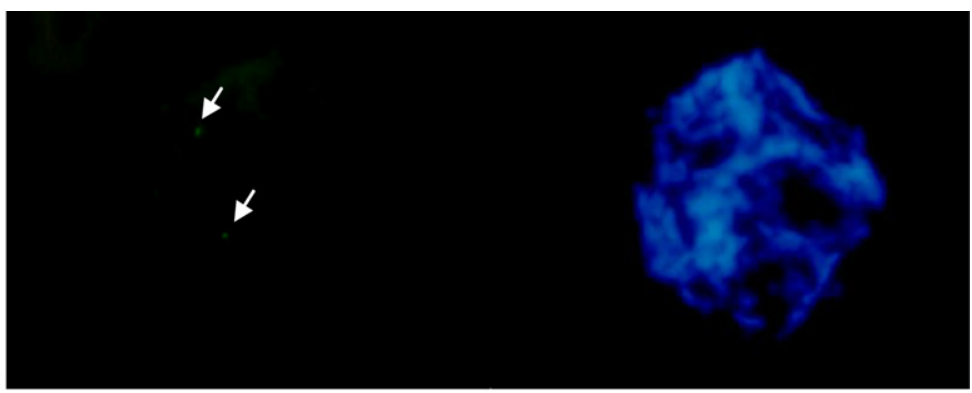

Panel B

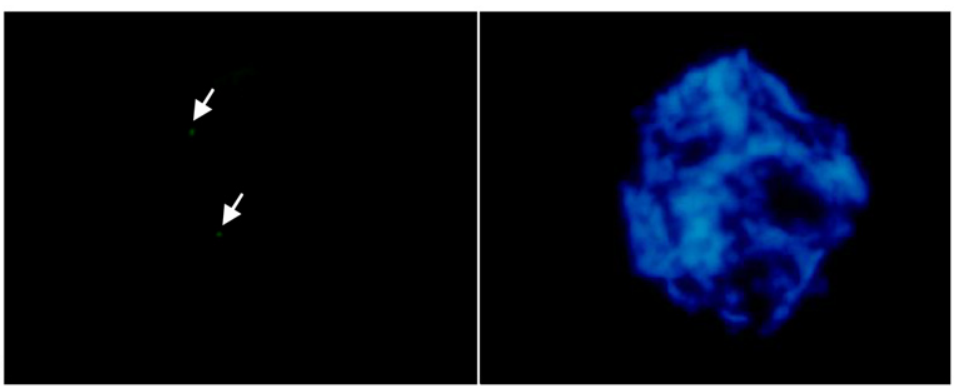

\section{Coupled image}

Supplementary Fig. 18. Blastomere with no transgene signal (the corresponding picture was not taken), and two control signals. (A) Microscopic images after the FISH procedure. (B) Same images as in (A) after background removal. In (A) and (B), the left panel shows a bovine chromosome 19 pericentromeric probe labeled with FITC (green signal); and the right panel, DAPI staining of chromosomes. (C) Image with the red and green signals coupled to the nuclei, generated with the image operations tool of Image Pro Plus or with similar tools from Photoshop, by superposition of the images shown in (B). 

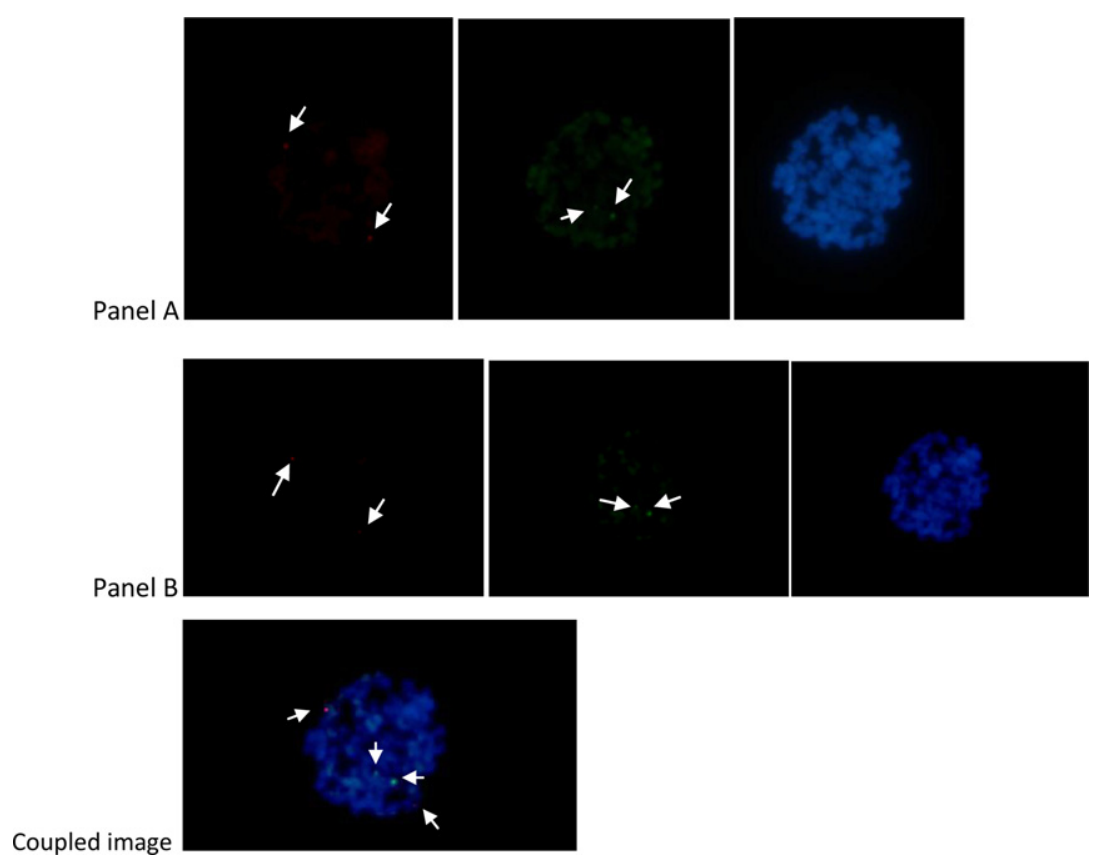

Supplementary Fig. 19. Blastomere with two transgene and two control signals. (A) Microscopic images after the FISH procedure. (B) Same images as in (A) after background removal. In (A) and (B), the left panel shows a $g f p$ rhodamine-labeled probe (red signal); the middle panel, a bovine chromosome 19 pericentromeric probe labeled with FITC (green signal); and right panel, DAPI staining of chromosomes. (C) Image with the red and green signals coupled to the nuclei, generated with the image operations tool of Image Pro Plus or with similar tools from Photoshop, by superposition of the images shown in (B). 


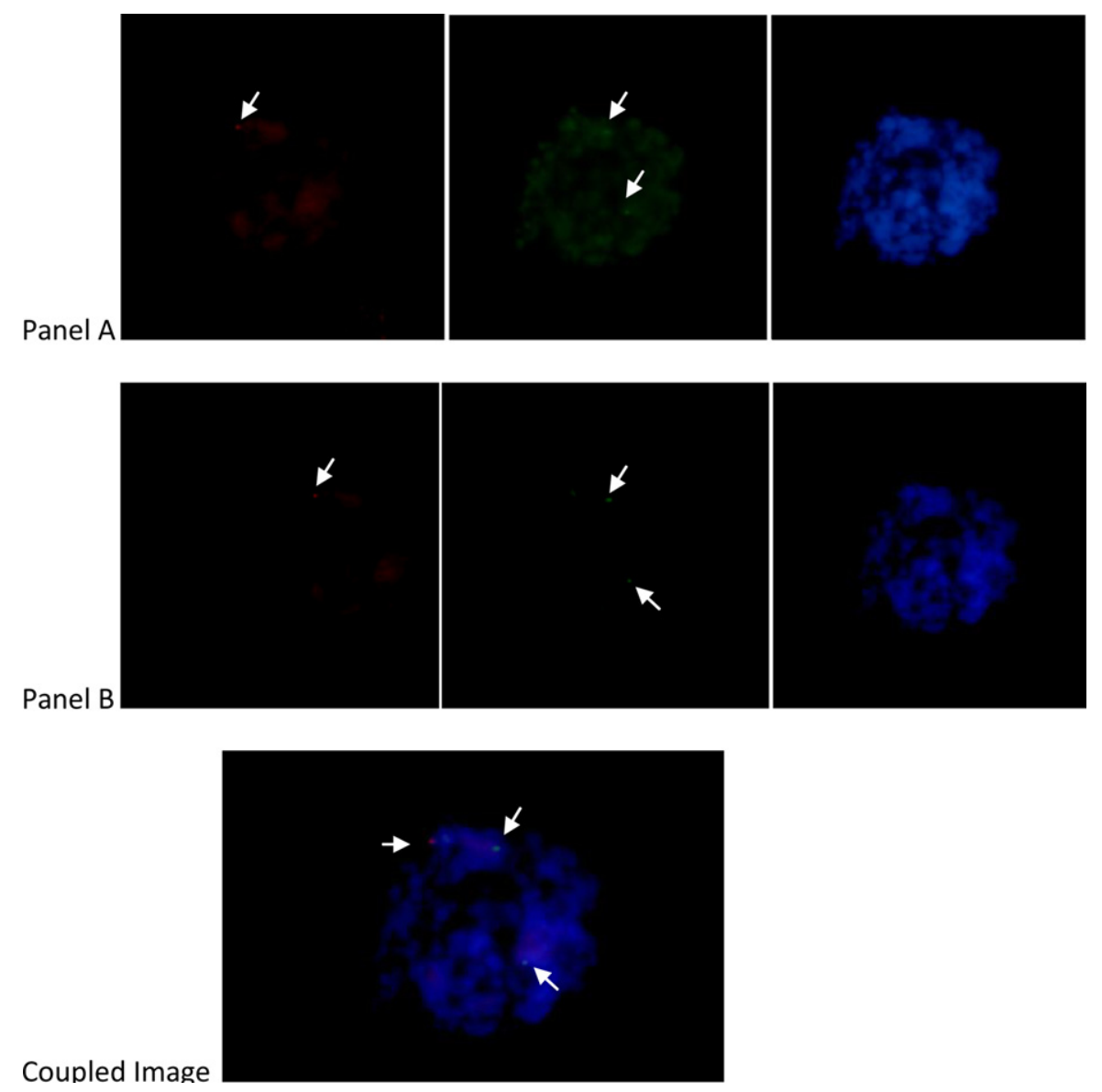

Supplementary Fig. 20. Blastomere with one transgene and two control signals. (A) Microscopic images after the FISH procedure. (B) Same images as in (A) after background removal. In (A) and (B), the left panel shows a gfp rhodamine-labeled probe (red signal); the middle panel, a bovine chromosome 19 pericentromeric probe labeled with FITC (green signal); and right panel, DAPI staining of chromosomes. (C) Image with the red and green signals coupled to the nuclei, generated with the image operations tool of Image Pro Plus or with similar tools from Photoshop, by superposition of the images shown in (B). 

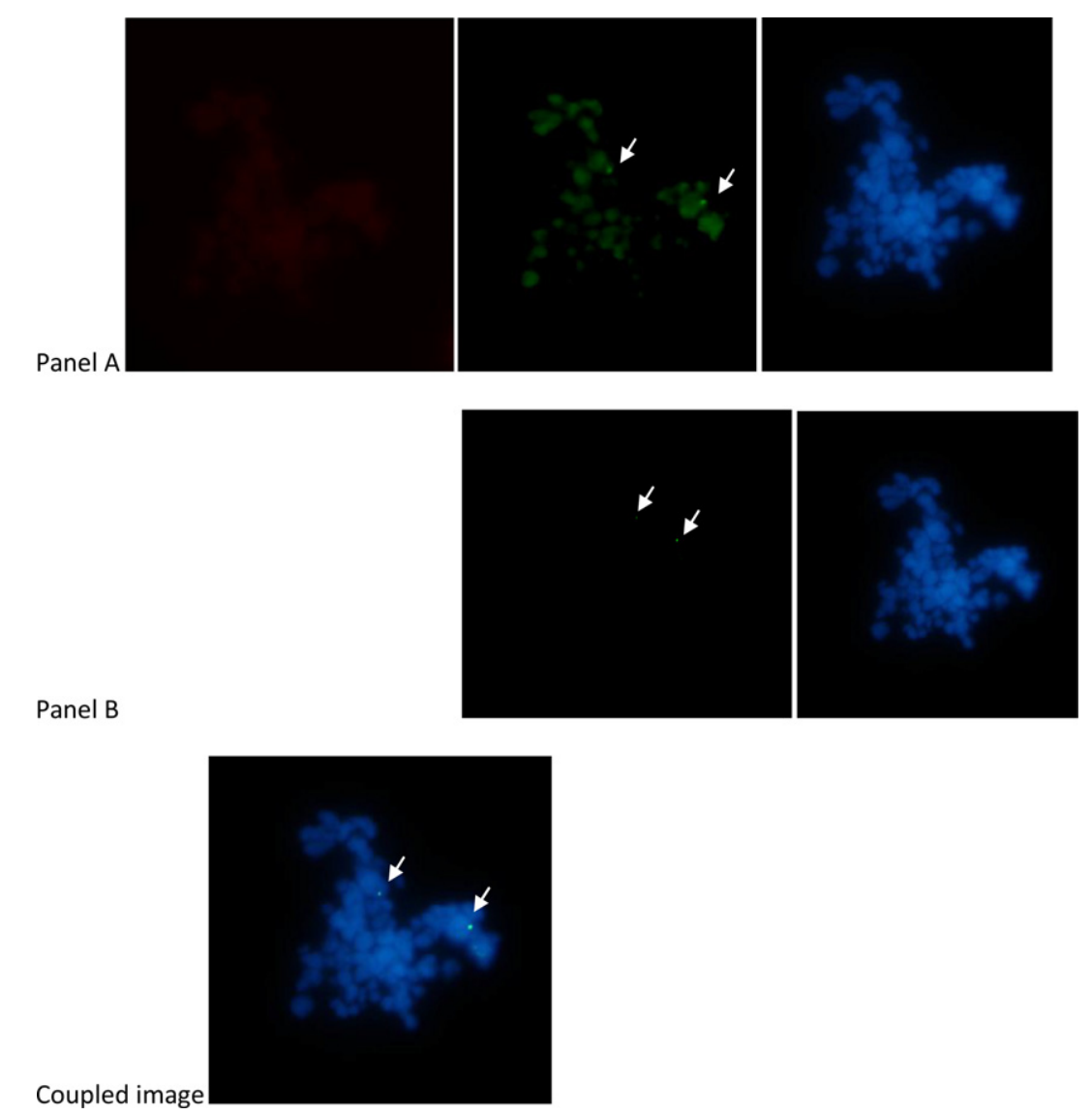

Supplementary Fig. 21. Blastomere with no transgene and two control signals. (A) Microscopic images after the FISH procedure. (B) Same images as in (A) after background removal. In (A), the left panel shows a gfp rhodamine-labeled probe (red signal); the middle panel, a bovine chromosome 19 pericentromeric probe labeled with FITC (green signal); and right panel, DAPI staining of chromosomes. In (B), the left pannel was not included as no gfp signal was detected. The middle and right pannels are equivalent to the ones shown in (A). (C) Image with the red and green signals coupled to the nuclei, generated with the image operations tool of Image Pro Plus or with similar tools from Photoshop, by superposition of the images shown in (B).
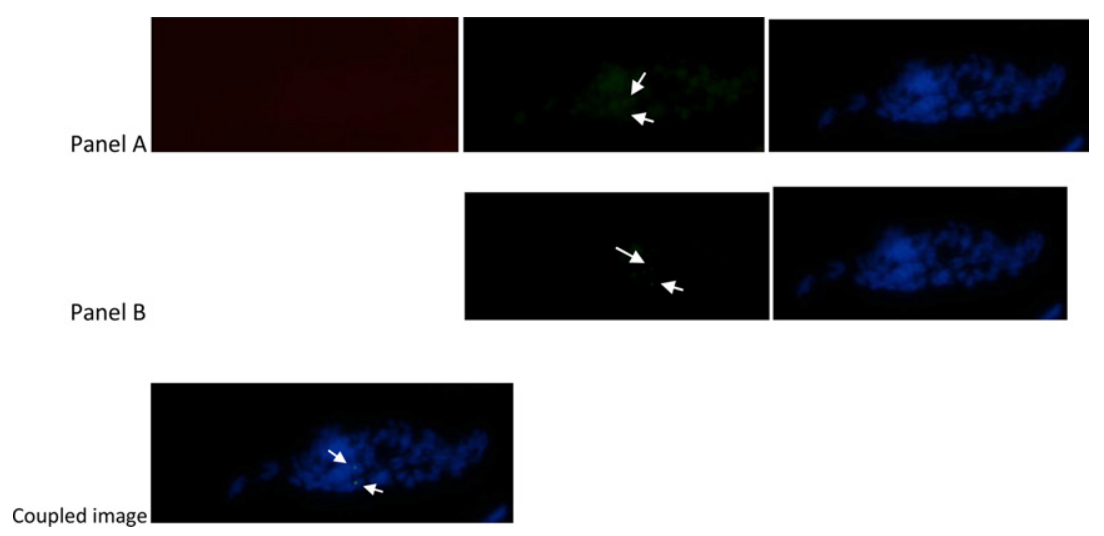

Supplementary Fig. 22. Blastomere with no transgene and two control signals. (A) Microscopic images after the FISH procedure. (B) Same images as in (A) after background removal. In (A), the left panel shows the bastomere under red light detection, a bovine chromosome 19 pericentromeric probe labeled with FITC (green signal); and right panel, DAPI staining of chromosomes. In (B), the left panel is omitted as no rhodamine signal was detected, the remaining pannels are the same as in (A). (C) Image with the red and green signals coupled to the nuclei, generated with the image operations tool of Image Pro Plus or with similar tools from Photoshop, by superposition of the images shown in (B). 

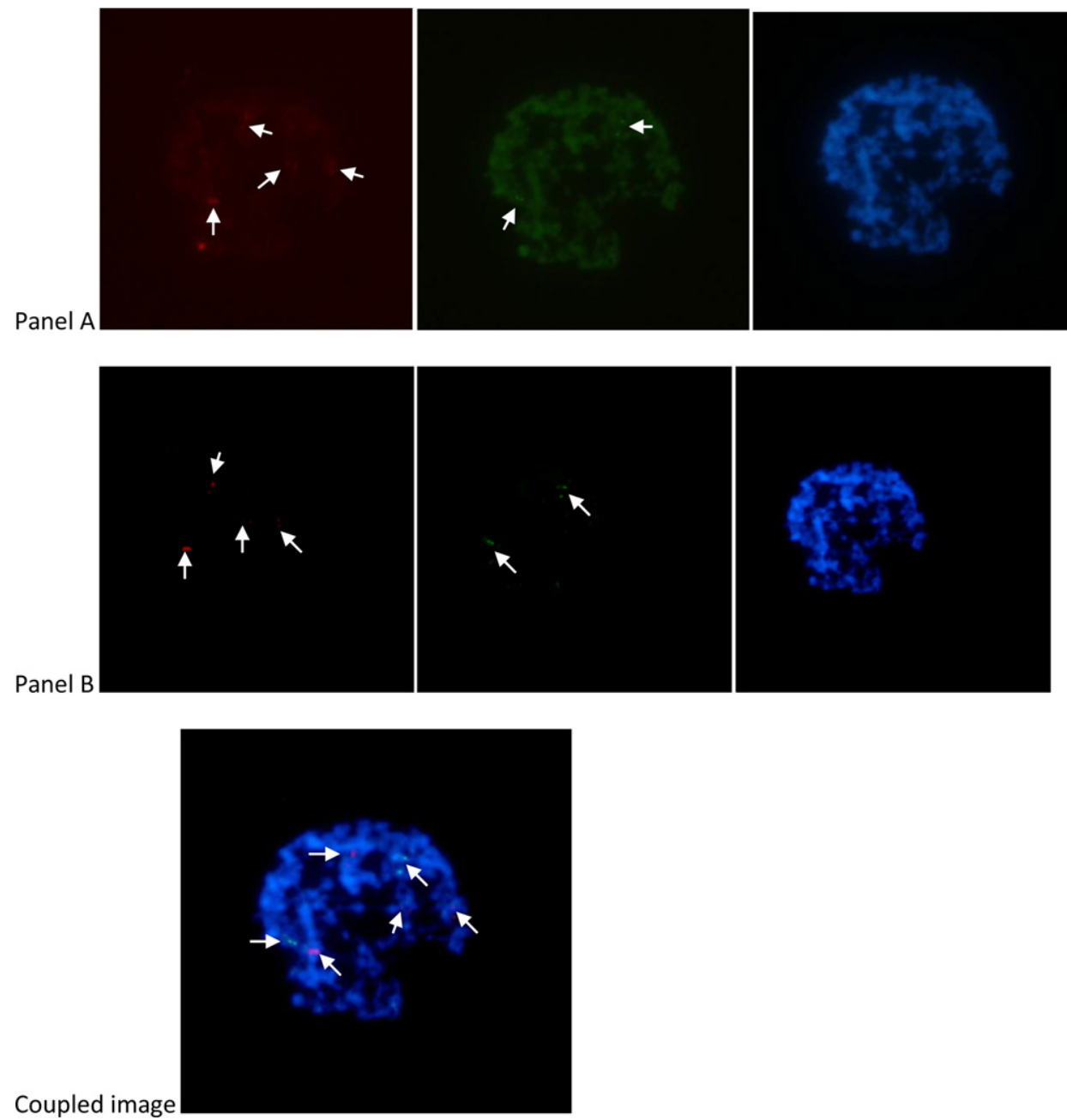

Supplementary Fig. 23. Blastomere with four transgene (in pairs) and two control signals (also in pairs). (A) Microscopic images after the FISH procedure. (B) Same images as in (A) after background removal. In (A) and (B), the left panel shows a gfp rhodamine-labeled probe (red signal); the middle panel, a bovine chromosome 19 pericentromeric probe labeled with FITC (green signal); and right panel, DAPI staining of chromosomes. (C) Image with the red and green signals coupled to the nuclei, generated with the image operations tool of Image Pro Plus or with similar tools from Photoshop, by superposition of the images shown in (B). 

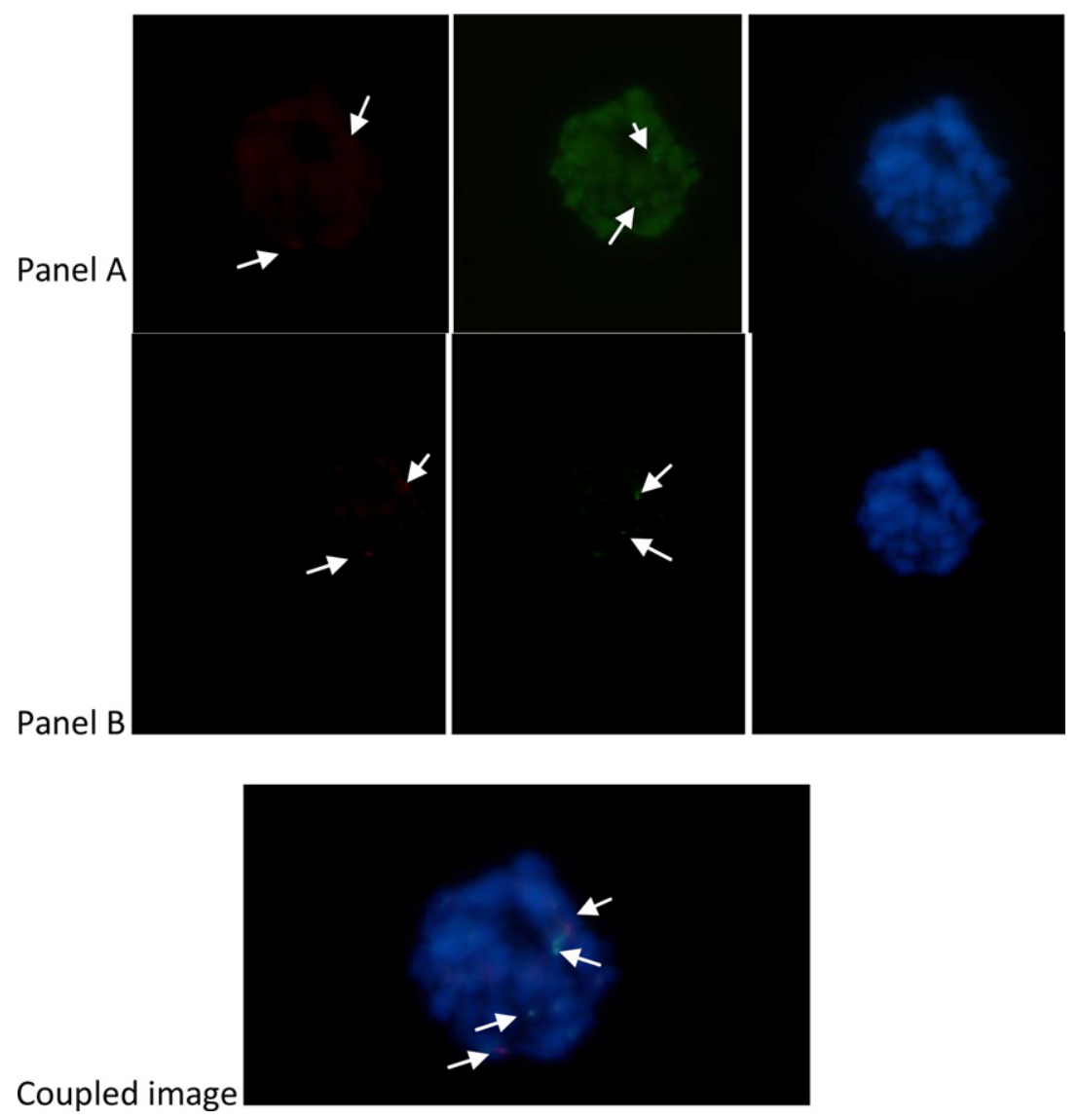

Supplementary Fig. 24. Blastomere with two transgene (not very clear in the picture) and two control signals. (A) Microscopic images after the FISH procedure. (B) Same images as in (A) after background removal. In (A) and (B), the left panel shows a gfp rhodamine-labeled probe (red signal); the middle panel, a bovine chromosome 19 pericentromeric probe labeled with FITC (green signal); and right panel, DAPI staining of chromosomes. (C) Image with the red and green signals coupled to the nuclei, generated with the image operations tool of Image Pro Plus or with similar tools from Photoshop, by superposition of the images shown in (B). 

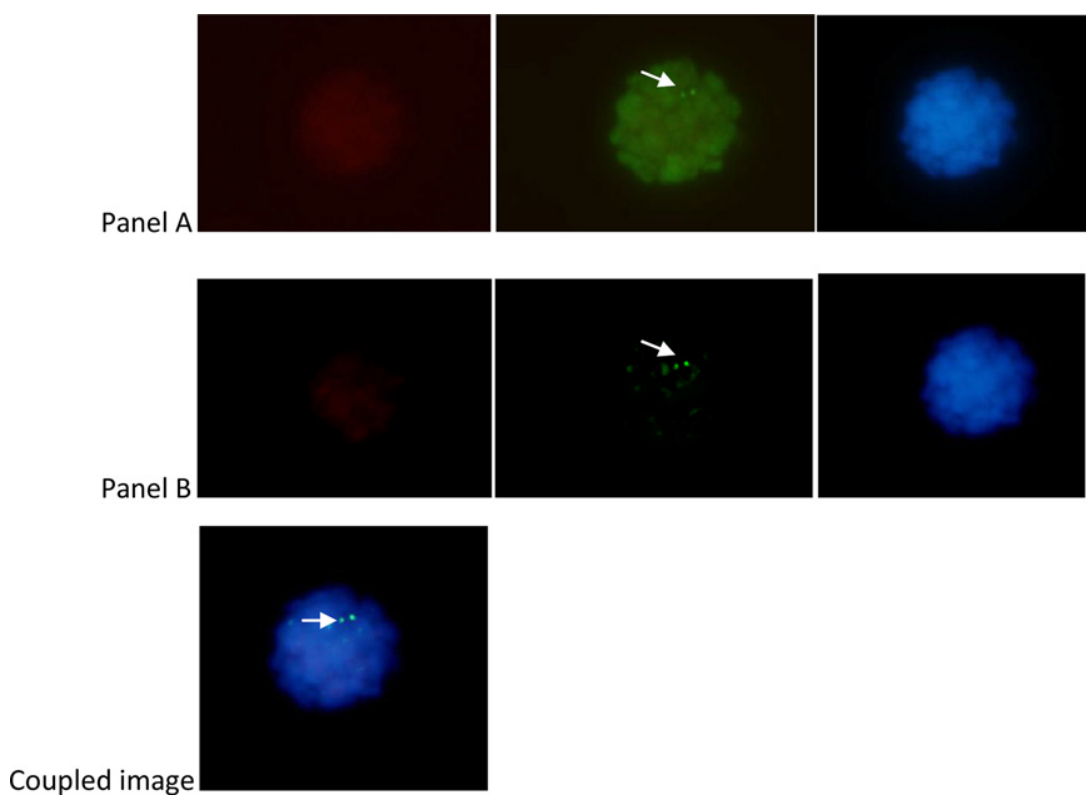

Supplementary Fig. 25. Blastomere with no transgene and one control signal (in a pair). (A) Microscopic images after the FISH procedure. (B) Same images as in (A) after background removal. In (A) and (B), the left panel shows a gfp rhodamine-labeled probe (red signal); the middle panel, a bovine chromosome 19 pericentromeric probe labeled with FITC (green signal); and right panel, DAPI staining of chromosomes. (C) Image with the red and green signals coupled to the nuclei, generated with the image operations tool of Image Pro Plus or with similar tools from Photoshop, by superposition of the images shown in (B). 

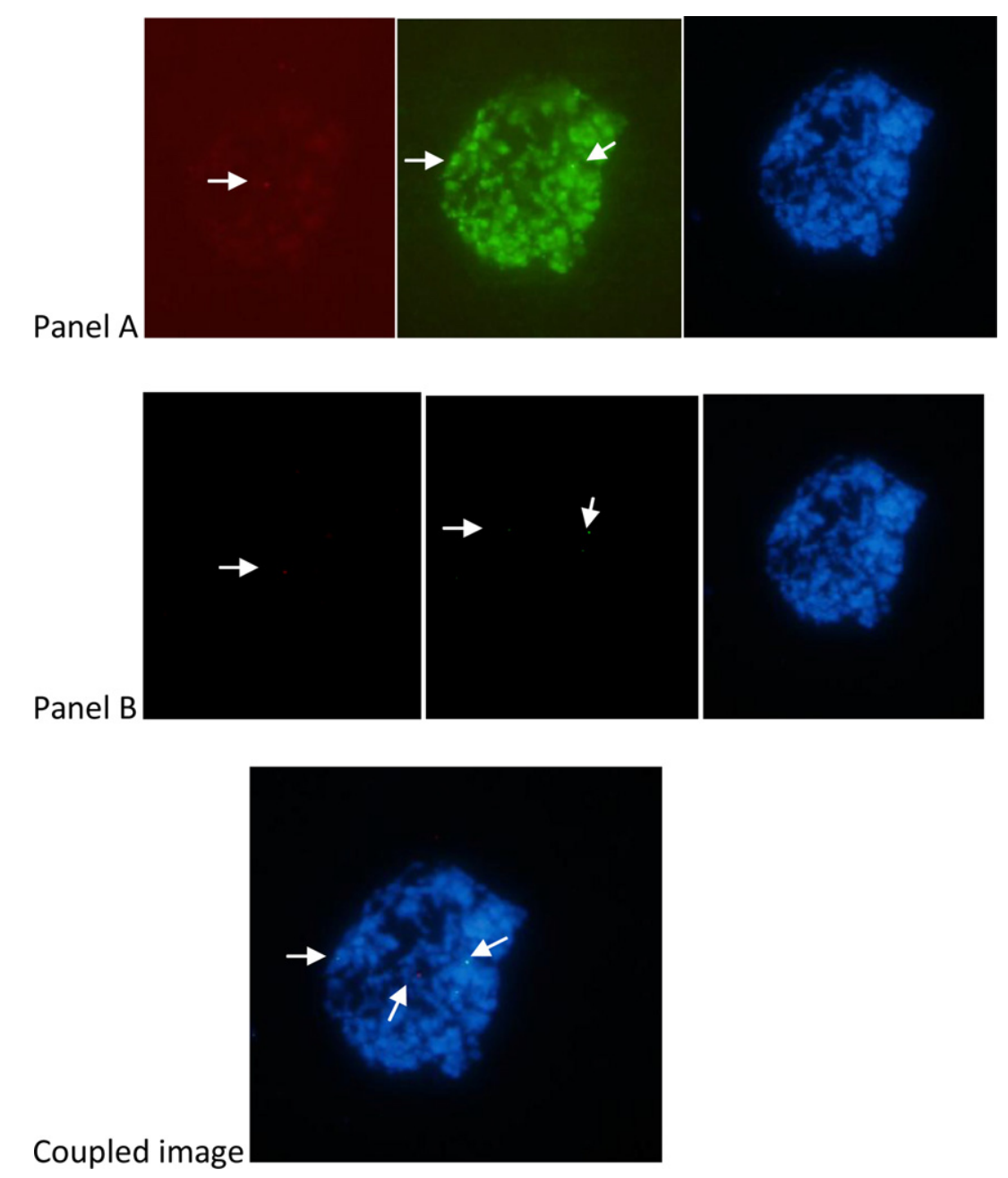

Supplementary Fig. 26. Blastomere with one transgene and two control signals. (A) Microscopic images after the FISH procedure. (B) Same images as in (A) after background removal. In (A) and (B), the left panel shows a gfp rhodamine-labeled probe (red signal); the middle panel, a bovine chromosome 19 pericentromeric probe labeled with FITC (green signal); and right panel, DAPI staining of chromosomes. (C) Image with the red and green signals coupled to the nuclei, generated with the image operations tool of Image Pro Plus or with similar tools from Photoshop, by superposition of the images shown in (B). 

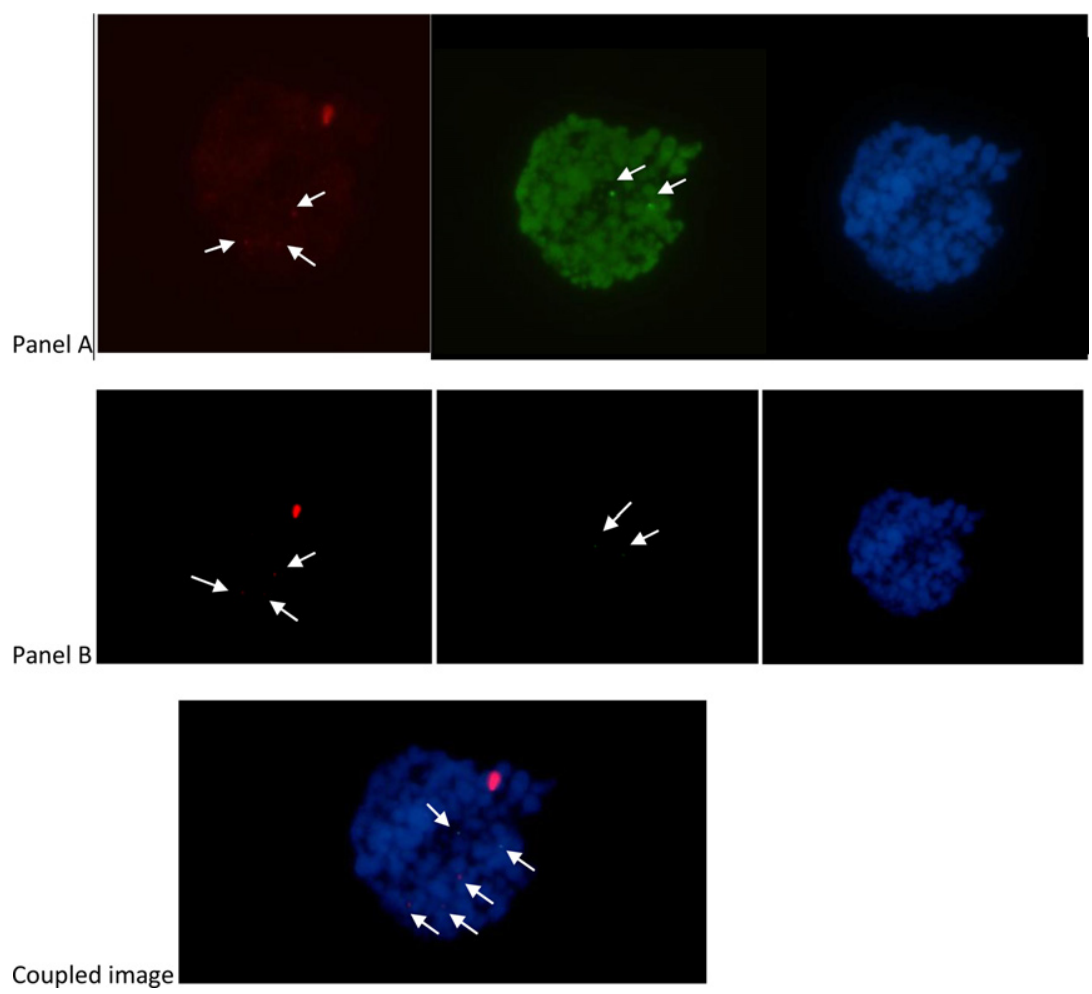

Supplementary Fig. 27. Blastomere with three transgene and two control signals. (A) Microscopic images after the FISH procedure. (B) Same images as in (A) after background removal. In (A) and (B), the left panel shows a gfp rhodamine-labeled probe (red signal); the middle panel, a bovine chromosome 19 pericentromeric probe labeled with FITC (green signal); and right panel, DAPI staining of chromosomes. (C) Image with the red and green signals coupled to the nuclei, generated with the image operations tool of Image Pro Plus or with similar tools from Photoshop, by superposition of the images shown in (B). 

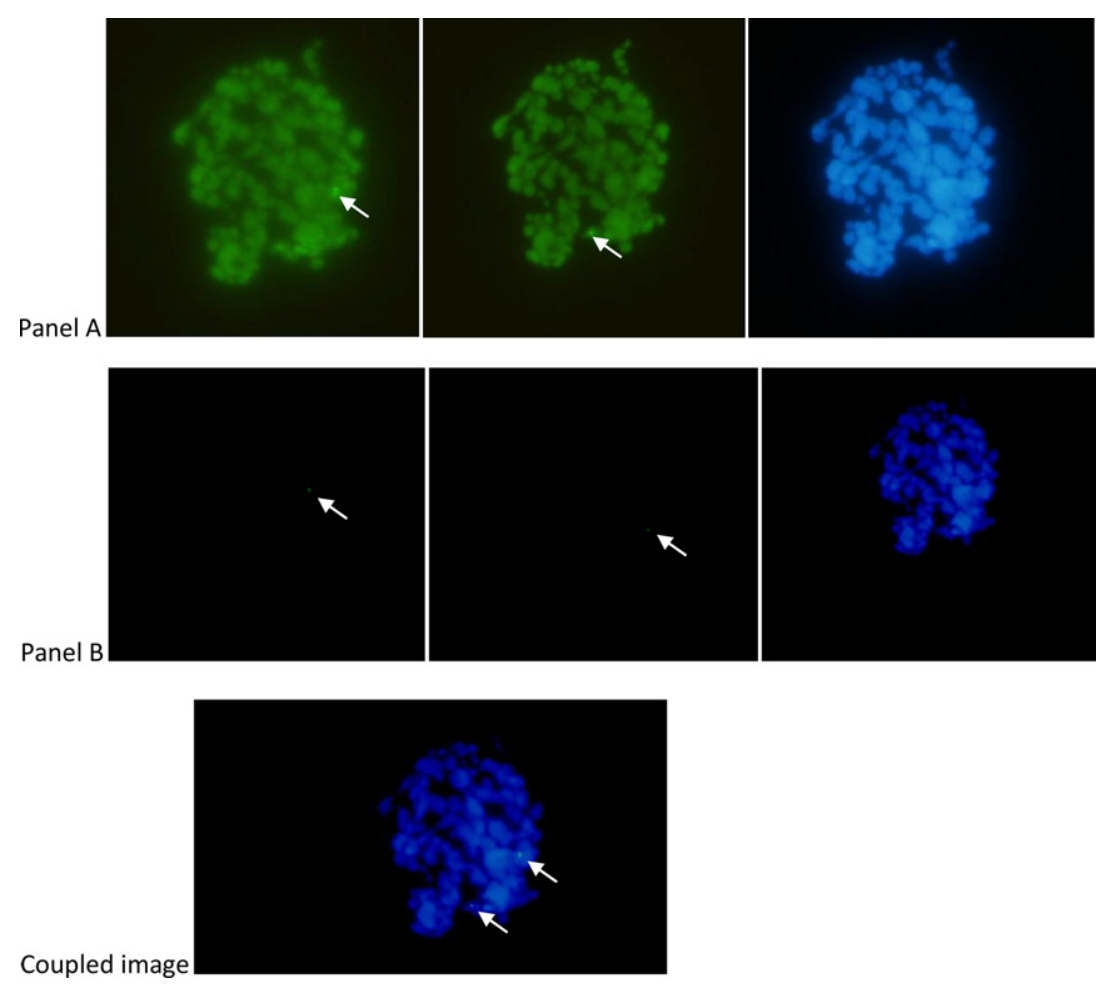

Supplementary Fig. 28. Blastomere with no transgene and two control signals in different layers. (A) Microscopic images after the FISH procedure. (B) Same images as in (A) after background removal. In (A) and (B), the left panel shows a gfp rhodamine-labeled probe (red signal); the middle panel, a bovine chromosome 19 pericentromeric probe labeled with FITC (green signal); and right panel, DAPI staining of chromosomes. (C) Image with the red and green signals coupled to the nuclei, generated with the image operations tool of Image Pro Plus or with similar tools from Photoshop, by superposition of the images shown in $(B)$. 


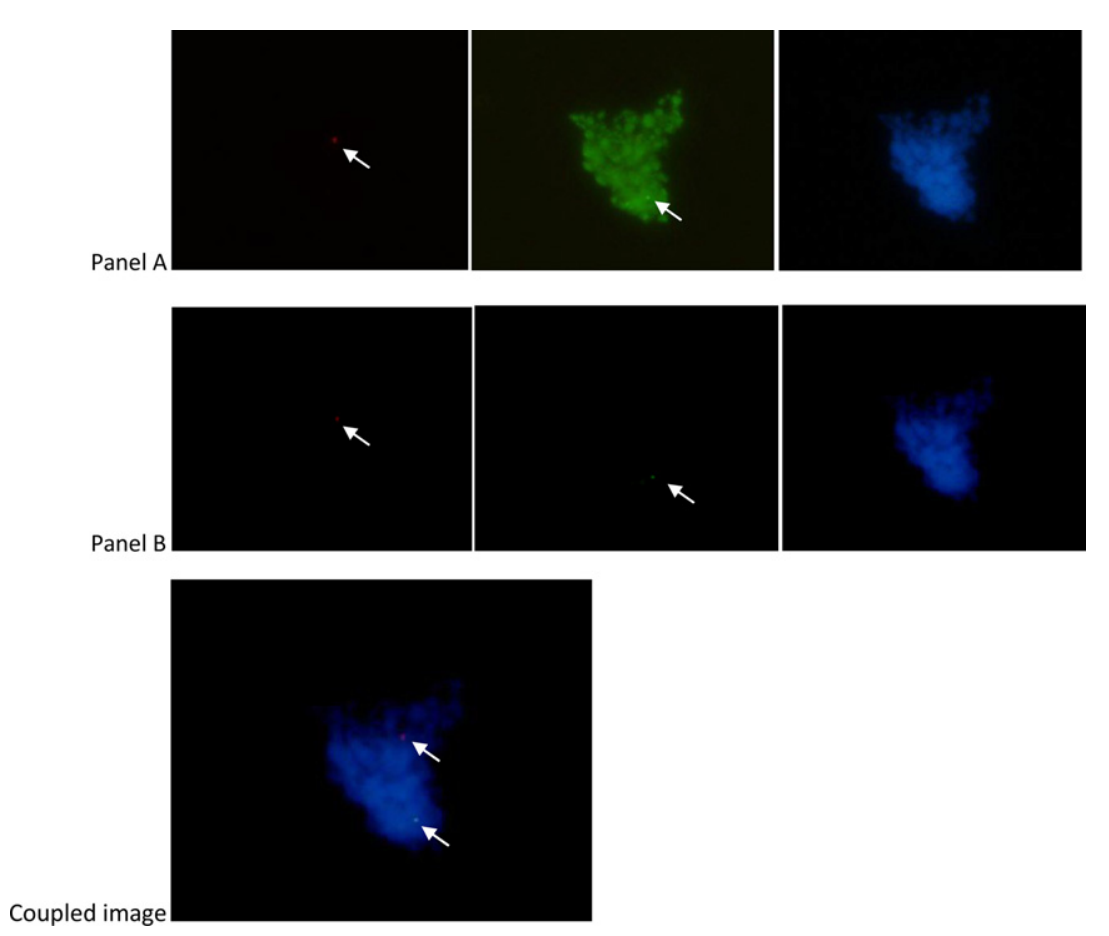

Supplementary Fig. 29. Blastomere with one transgene and one control signal. (A) Microscopic images after the FISH procedure. (B) Same images as in (A) after background removal. In (A) and (B), the left panel shows a $\mathrm{g} f$ rhodamine-labeled probe (red signal); the middle panel, a bovine chromosome 19 pericentromeric probe labeled with FITC (green signal); and right panel, DAPI staining of chromosomes. (C) Image with the red and green signals coupled to the nuclei, generated with the image operations tool of Image Pro Plus or with similar tools from Photoshop, by superposition of the images shown in (B). 


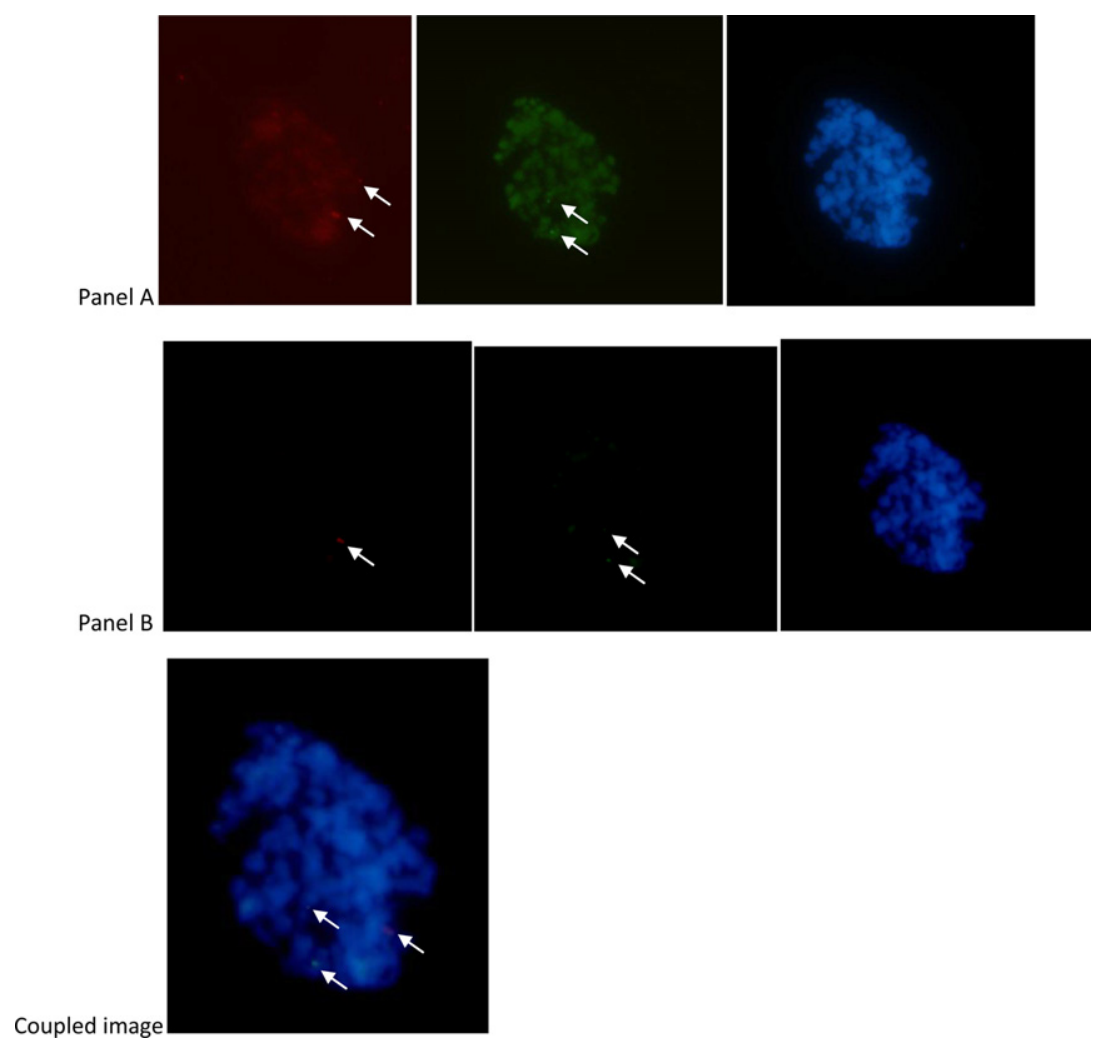

Supplementary Fig. 30. Blastomere with two transgene (one lost after background removal) and two control signals. (A) Microscopic images after the FISH procedure. (B) Same images as in (A) after background removal. In (A) and (B), the left panel shows a $g f p$ rhodamine-labeled probe (red signal); the middle panel, a bovine chromosome 19 pericentromeric probe labeled with FITC (green signal); and right panel, DAPI staining of chromosomes. (C) Image with the red and green signals coupled to the nuclei, generated with the image operations tool of Image Pro Plus or with similar tools from Photoshop, by superposition of the images shown in (B). 